\title{
Sistema de Acreditación Universitaria en Chile: ¿Cuánto hemos avanzado?
}

\author{
University Accreditation System in Chile: What have we Come?
}

Sistema de aprovação no Chile: quanto temos avançado?

\author{
Víctor Cancino C., ${ }^{a}$ Rodolfo Schmal S. ${ }^{b}$ \\ anstituto de Investigación y Desarrollo Educacional, U. de Talca. Telf.: 56-71-200256. Correo electrónico: \\ vcancino@utalca.cl
}

bFacultad de Ciencias Empresariales, U. de Talca. Telf.: 56-71-200354 U. Correo electrónico: rschmal@utalca.cl

\begin{abstract}
RESUMEN
Hasta mediados de los 80 , el sistema universitario chileno puede ser considerado relativamente estático y pequeño, sin embargo, a partir de la reforma de 1981, empieza a transformarse en uno de gran dinamismo y alto crecimiento. Producto de esta expansión, en 1999 se sientan las bases del sistema de acreditación de educación superior en Chile, a partir de la creación de la Comisión Nacional de Acreditación de Pregrado (CNAP) y la Comisión Nacional de Acreditación de Postgrado (CONAP), instituciones que posteriormente se fusionan conformando la Comisión Nacional de Acreditación (CNA). Este trabajo analiza el estado actual de los procesos de acreditación universitaria en Chile -en el ámbito institucional, de programas de pregrado y postgrado- comparando un conjunto de indicadores según tipologías convencionales del sistema universitario nacional. El estudio es de tipo descriptivo sustentado en información de bases de datos oficiales de la CNA y el Consejo Nacional de Educación, sobre los resultados de los procesos de acreditación universitaria e indicadores institucionales. Los hallazgos dan cuenta de la diversidad institucional imperante y, especialmente, de la diversidad cualitativa en la oferta educacional universitaria chilena, tanto de pregrado como de posgrado.
\end{abstract}

Palabras clave: acreditación universitaria, indicadores de calidad, aseguramiento de la calidad.

\begin{abstract}
Until the mid 80's, the Chilean university system can be considered relatively static and small, however, after the 1981 reform, it became highly dynamic and presents a huge growth. As a consequence of this expansion, in 1999 the foundations of the accreditation system of higher education in Chile were built, from the creation of the National Undergraduate Accreditation Commission (CNAP its acronym in Spanish) and the National Commission on Accreditation of Graduate Studies (CONAP). Subsequently, these two mentioned institutions merged forming the National Accreditation Commission (CNA). This paper analyzes the current state of the university accreditation processes in Chile - at the institutional level of undergraduate and graduate programs - comparing a set of indicators according to conventional typology provided by the national university system. The study is descriptive, based on information from official databases of the CNA and the National Education Council about the results of university accreditation process and institutional indicators. The findings give account of the prevailing institutional diversity, and especially the qualitative diversity in the Chilean university educational offer, both undergraduate and graduate students.
\end{abstract}

Key words: university accreditation, quality indicators, quality assurance.

\section{RESUMO}

Até meados dos anos 80, o sistema universitário chileno pode ser considerado relativamente estático e pequeno; no entanto, desde a reforma de 1981, começa a transformar-se em um sistema de grande dinamismo e acelerado crescimento. Resultado de essa expansão, em 1999, assentam-se as bases do sistema de aprovação da Educação Superior no Chile, a partir da criação da Comisión Nacional de Acreditación de Pregrado (CNAP) e da Comisión Nacional de Acreditación de Postgrado (CONAP), instituições que, posteriormente, se fundiram formando, então, a Comisión Nacional de Acreditación (CNA). Analisa-se o estado atual dos processos de aprovação universitária no Chile - no âmbito institucional dos cursos de graduação e pós-graduação - comparando-se um conjunto de indicadores consoante tipologias convencionais do sistema universitário nacional. O estudo é descritivo e baseado em informações da base de dados oficiais do CNA e do Conselho Nacional de Educação sobre os resultados dos processos de aprovação universitária e indicadores institucionais. Achados sustentam a diversidade institucional vigente e, especialmente, a diversidade qualitativa na oferta educacional universitária, tanto na graduação como na pós-graduação.

Palavras chave: aprovação universitária, indicadores de qualidade, garantia de qualidade. 


\section{INTRODUCCIÓN}

El sistema universitario chileno ha sufrido una profunda transformación en los últimos 30 años en términos de su cobertura, masificación, financiamiento y normativas, como consecuencia de las políticas de liberalización implementadas a inicio de los 80 . A propósito de ello, en consonancia con tendencias internacionales en la materia, ha sido necesaria la introducción de mecanismos de regulación destinados al aseguramiento de la calidad universitaria. Este trabajo se inserta en este último ámbito, centrándose en las características y el estado en que se encuentra los procesos de acreditación vigentes en Chile.

Para estos efectos, el trabajo se inicia con una presentación de la evolución que ha experimentado el sistema universitario chileno, seguido por las características centrales del sistema de acreditación universitaria existente. Posteriormente, se esquematiza cuantitativamente el estado de la acreditación de las universidades a nivel institucional y de sus programas de pregrado y postgrado, para finalizar con las conclusiones que se desprenden de la información obtenida al año 2010.

El trabajo busca mostrar el estado de avance de la acreditación universitaria en Chile, sistema que emerge a raíz de la existencia durante dos décadas, de un mercado universitario con altos grados de desregulación, que llevó a la creación de un número sustantivo de universidades, lo que contribuyó al incremento de la matrícula de educación terciaría, mediante la configuración de una oferta cualitativamente diversa que nace de instituciones con grados de desarrollo muy dispares. En este escenario, la acreditación universitaria surge como una alternativa para transparentar información sobre el sistema y contar con antecedentes confiables y comparables, considerando la relevancia de la educación universitaria para el desarrollo del país, las familias y la sociedad en su conjunto, dotando de instrumentos al Estado (políticas públicas) y la sociedad (accountability), para regular, orientar y conducir el sistema universitario.

\section{LA EVOLUCIÓN DEL SISTEMA UNIVERSITARIO CHILENO}

El sistema universitario chileno en 30 años ha transitado desde un periodo de alta rigidez, a uno actualmente de extrema flexibilidad, siendo posible distinguir cuatro fases de evolución que han configurado el presente escenario. La primera etapa se extiende por más de 100 años, desde la creación de la primera universidad en 1842 hasta 1980, período en el que se crean solo 8 universidades, dos de ellas estatales y seis privadas. ${ }^{1}$ Estas últimas fueron consideradas como universidades de carácter público, operando desde sus inicios con financiamiento estatal, por su cooperación con la función educacional del Estado. La última universidad creada en este periodo fue la Universidad Católica del Norte, fundada en 1956 (inicialmente como Universidad del Norte, y desde 1964 con su nombre actual), lo que significa que durante los últimos 25 años de esta etapa no nacen nuevas instituciones. En esta etapa el sistema universitario chileno puede

Las instituciones estatales Universidad de Chile y Universidad Técnica del Estado (hoy Universidad de Santiago) y las 6 restantes son universidades privadas, siendo estas: Pontificia Universidad Católica de Chile, de Concepción, Católica de Valparaíso, Técnica Federico Santa María, Austral de Chile y Católica del Norte. 
ser considerado pequeño y caracterizado por un bajo nivel de cobertura respecto de la población en edad correspondiente, algo que fue propio de los sistemas educativos en América Latina hasta esa época.

La segunda fase se inicia en 1981 con la promulgación durante el Régimen Militar del decreto con fuerza de ley $\mathrm{N}^{\circ} 2$ del 3 de enero de 1981, mediante el cual se inicia un camino marcado por la fragmentación de las universidades estatales, a quienes se les despoja de sus sedes regionales, para dar origen a nuevas universidades estatales, ${ }^{2}$ iniciando al mismo tiempo la liberalización y creación de nuevas universidades privadas, lo que llevó a que a fines de 1989 se hubiesen creado 11 universidades privadas, ${ }^{3}$ llegando a un total 33 instituciones universitarias en funcionamiento (Cruz-Coke, 2004).

Estos cambios iniciales aumentaron su intensidad al acercarse el término del Régimen Militar, lo que implicó que, según Bernasconi y Rojas (2003: 19), "a partir de marzo de 1990 el proceso se aceleró aún más; sólo entre principios de enero y el 7 de marzo de 1990 se autorizaron 18 universidades y 23 institutos profesionales, y seis instituciones quedaron en trámite". Será también, al término del gobierno militar, donde se resuelve dictar la Ley $N^{\circ} 18.962$-Ley Orgánica Constitucional de Enseñanza (LOCE)- que modifica las bases del sistema educativo nacional, estando en vigencia y sin grandes modificaciones durante casi 20 años, desde 1990 a 2009. Esta Ley crea el Consejo Superior de Educación (CSE), organismo destinado a supervigilar el sistema educativo nacional y el universitario en particular.

La tercera fase de desarrollo, entre 1990 y 1999, al amparo de la LOCE, se caracteriza por el crecimiento explosivo de universidades privadas y la continuidad de una tendencia de fragmentación que lleva a la Pontificia Universidad Católica de Chile, en 1991, a que sus sedes regionales pasen a desarrollarse autónomamente, creándose así tres nuevas universidades católicas. ${ }^{4}$ Durante esta etapa, en un periodo de 5 años, el sistema más que se duplica en cantidad, creándose 39 universidades, llegando a un total de 70 instituciones en 1993, situación que se mantiene hasta el año 1995 y empieza a declinar lentamente hasta 1999 llegando a un total de 65 universidades en funcionamiento (Bernasconi y Rojas, 2003). Para Zapata (2004: 143), este crecimiento es consecuencia de un esquema de educación superior caracterizado por la ausencia de regulación, razón por la cual "la acreditación ha surgido como una alternativa de control moderna, flexible, acorde a las características de nuestras instituciones, y de rango o clase internacional".

Finalmente, la cuarta fase, desde 2000 a la fecha, se ha caracterizado por un proceso de creación, fusión y desaparición de universidades privadas hasta llegar en la actualidad a un sistema con 60 universidades ( 35 privadas), manteniendo sin modificación la cantidad de universidades estatales y privadas tradicionales (Tabla 1). Esta es una etapa donde se inicia la implementación de un proceso de regulación como expresión de la preocupación por el aseguramiento de la calidad en la educación superior.

2 Las nuevas universidades estatales creadas fueron: Universidad de Antofagasta, de Atacama, de La Serena, de Tarapacá, Arturo Prat, de Valparaíso, de Playa Ancha, Metropolitana de Ciencias de la Educación, de Talca, del Bío-Bío, de la Frontera, de Magallanes, de Los Lagos (creada en 1993, desde 1981 como IP de Osorno) y Tecnológica Metropolitana (creada en 1993, existiendo desde 1981 como IP de Santiago).

3 Las universidades privadas fueron Universidad Diego Portales, Gabriela Mistral, Central, Mayor, Finis Terrae, La República, Bolivariana, Andrés Bello, Adolfo Ibáñez, Academia de Humanismo Cristiano y Las Américas.

4 Las Universidades Católica de Temuco, Católica de la Santísima Concepción y Católica del Maule. 
Tabla 1. $\mathrm{N}^{\circ}$ de universidades en funcionamiento por periodo

\begin{tabular}{|c|c|c|c|c|}
\hline Fase & $\mathrm{N}^{\circ} \mathrm{U}$. Estatales & $\begin{array}{c}\mathrm{N}^{\circ} \text { U. Privadas } \\
\text { Tradicionales }\end{array}$ & $\mathrm{N}^{\circ}$ U. Privadas & $\begin{array}{c}\text { Total } \\
\text { Universidades }\end{array}$ \\
\hline $1842-1980$ & 2 & 6 & 0 & 8 \\
\hline $1981-1989$ & 14 & 6 & 11 & 31 \\
\hline $1990-1999$ & 16 & 9 & 45 & 70 \\
\hline $2000-2010$ & 16 & 9 & 35 & 60 \\
\hline
\end{tabular}

Fuente: Elaboración propia a partir de Bernasconi y Rojas (2003), Cruz-Coke(2004), Ugarte (2010) y CNED (2010).

Si bien la primera fase, hasta 1980, se caracteriza por su estabilidad. En la segunda se inicia un proceso gradual de expansión que se expresa en la creación de aproximadamente dos universidades por año. Este crecimiento se acelera en la tercera fase (1990-1999) más que duplicando el número de instituciones, expansión que se revierte en la cuarta y última fase, donde se observa una disminución paulatina de universidades, lo que revela cambios orientados a regular un mercado de educación superior, hasta entonces, con altos grados de desregulación.

Esta dinámica refleja una tendencia -que se cree- proseguirá en los próximos años, actuando la acreditación como mecanismo de regulación del sistema universitario. A pesar que la acreditación institucional es un proceso de carácter voluntario, un número creciente de universidades, así como programas de pregrado y postgrado, han optado por ello: 53 de las 60 universidades se han sometido a acreditación ante la Comisión Nacional de Acreditación (CNA, 2010; Ugarte, 2010).

En síntesis, la evolución del sistema universitario chileno ha implicado pasar desde un sistema estático compuesto solo por ocho universidades, hacia un sistema más abierto y diversificado, con dos universidades públicas redimensionadas y un número importante de universidades estatales localizadas en regiones (16 universidades), con universidades católicas desgajadas de la Pontificia Universidad Católica de Chile y universidades privadas tradicionales (9 instituciones). Todas las instituciones mencionadas están agrupadas en el Consejo de Rectores de Universidades de Chile $(\mathrm{CRUCH})^{5}$ y un número significativo de nuevas universidades privadas (35 en la actualidad).

\section{EL SISTEMA DE ACREDITACIÓN UNIVERSITARIA EN CHILE}

El dinamismo y la complejidad que caracterizó al sistema universitario chileno en la década de los 90, condujo a fines de esta década a la instalación de las bases del sistema de acreditación de educación superior, a partir de la creación de la Comisión Nacional de Acreditación de Pregrado (CNAP) y la Comisión Nacional de Acreditación de Postgrado (CONAP), mediante los decretos supremos $\mathrm{N}^{\circ} 59$ y N²25 del Ministerio de Educación,

$5 \quad$ Organismo fundado en 1954, bajo la Ley No 11.575 (artículo 36, letra c), como un organismo de coordinación de la labor universitaria de la nación, y que agrupa actualmente 25 universidades, las 16 universidades estatales y 9 universidades privadas tradicionales. 
que establecen las "normas sobre acreditación experimental y voluntaria de programas de pregrado y de postgrado" (Espinoza et al., 2006: 16). Este proceso conduce en el año 2003 a que se dé inicio a una experiencia piloto de acreditación donde fueron sometidas a evaluación 13 instituciones, ${ }^{6}$ las que "en su mayoría ya contaban con experiencias previas de evaluación” Zapata (2004: p.153).

El camino recorrido llevó en 2006 a promulgar la Ley $\mathrm{N}^{\circ} 20.129$ de Aseguramiento de la Calidad de la Educación Superior, la que da origen al sistema de acreditación vigente en Chile. La normativa asociada a la ley deja bajo la responsabilidad de la Comisión Nacional de Acreditación (CNA), la implementación de los procesos de acreditación institucional y de los programas de pre y postgrado. En el caso de estos últimos, la ley otorga facultades a agencias de acreditación privadas, para que bajo la supervisión de la CNA lleven a cabo procesos de acreditación.

En la actualidad, desde la dictación de la Ley General de Educación (LGE) a inicios del año 2010, la regulación de la educación superior quedó en manos del Consejo Nacional de Educación (CNED), heredero legal del Consejo Superior de Educación, entidad responsable del Sistema de Aseguramiento de la Calidad de la Educación, sistema que tiene los siguientes componentes: ${ }^{7}$

a) Información: identificar, recolectar y difundir los antecedentes necesarios para la gestión del sistema, y la información pública.

b) Licenciamiento: a realizar en conformidad a lo dispuesto en la ley $\mathrm{N}^{\circ} 18.962$, Orgánica Constitucional de Enseñanza.

c) Acreditación institucional: supervisar el proceso de análisis de los mecanismos existentes, junto a su aplicación y resultados al interior de las instituciones autónomas de educación superior para asegurar su calidad.

d) Acreditación de carreras o programas: supervisar el proceso de verificación de la calidad de las carreras o programas ofrecidos por las instituciones autónomas de educación superior, en función de sus propósitos declarados y de los criterios establecidos por las respectivas comunidades académicas y profesionales.

Los actores principales de este sistema son:

- El Consejo Nacional de Educación (CNED)

- La Comisión Nacional de Acreditación (CNA)

- El Ministerio de Educación, a través de su División de Educación Superior (MINEDUC)

El Consejo Nacional de Educación tienen un rol relevante en el Sistema Nacional de Aseguramiento de la Calidad de la Educación Superior, en funciones de información y acreditación (sea esta institucional o de programas), sin embargo, la función en la cual posee un papel prioritario es aquella referida al proceso de licenciamiento de las instituciones de educación superior. Según lo establece la LGE (artículo 97), este licenciamiento "comprende la aprobación del proyecto institucional y el proceso que permite evaluar el avance y concreción del proyecto educativo de la nueva entidad, a través de variables significativas de su desarrollo, tales como docentes, didácticas, técnico-pedagógicas, programas de estudios, físicos y de infraestructura, así como los recursos económicos y financieros necesarios para otorgar los grados académicos y los títulos de que se trate."

$6 \quad$ El grupo estaba conformado por 11 universidades, 8 pertenecientes al Consejo de Rectores y 3 privadas, además de 2 institutos profesionales (Mineduc, s/f)

Información disponible en www.cned.cl 
El proceso de licenciamiento es una etapa previa al proceso de acreditación y comprende tres fases fundamentales: (i) Aprobación del proyecto institucional, (ii) Verificación periódica del cumplimiento de los criterios de evaluación del Consejo (periodo que se puede extender entre un 6 y 11 años) y finalmente (iii) la autonomía institucional, estatus que faculta a las universidades para dictar libremente programas de pre y postgrado, y que les permite someterse a un proceso de acreditación institucional.

Según lo establece la normativa vigente (Art. 15), los procesos de acreditación son voluntarios (salvo para carreras de educación y medicina), y si bien solo la acreditación institucional establece que las etapas formales corresponden a evaluación interna, evaluación externa y juicio de acreditación, estas mismas han sido incorporadas en forma implícita como parte de los procesos de acreditación de los programas de pre y postgrado. Estas etapas, según lo establece la CNA, en lo fundamental corresponden a:

a) Autoevaluación Interna: Proceso analítico que consulta diferentes fuentes, tanto internas como externas a la institución, que identifica los mecanismos de autorregulación existentes, las fortalezas y debilidades de la institución con relación a ellos, buscando verificar el cumplimiento oportuno y satisfactorio de los objetivos y propósitos definidos en su misión y fines institucionales.

b) Evaluación Externa: Proceso tendiente a certificar que la institución cuenta con las condiciones necesarias para asegurar un avance sistemático hacia el logro de sus propósitos declarados, a partir de la evaluación de las políticas y mecanismos de autorregulación vigentes en ella, proceso que se lleva a cabo mediante la visita de pares evaluadores.

c) Pronunciamiento de la Comisión: Corresponde al juicio emitido por la comisión de acreditación respectiva en base a la ponderación de los antecedentes recabados, mediante el cual se determina acreditar o no acreditar a la institución (programa de pregrado o postgrado), en virtud de la existencia y nivel de desarrollo de sus políticas y mecanismos de aseguramiento de la calidad.

\section{SITUACIÓN DE LA ACREDITACIÓN INSTITUCIONAL A NIVEL UNIVERSITARIO}

La situación actual de la acreditación institucional, considerando las 60 universidades existentes en 2010, muestra que un $73 \%$ de las universidades se encuentran acreditadas (en primera o segunda instancia), un $17 \%$ no ha logrado la acreditación, ${ }^{8}$ un $2 \%$ se encuentra en proceso ${ }^{9}$ y un $8 \%$ de las universidades no han sido sometidas a acreditación, por no cumplir con los requisitos establecidos en la legislación vigente o por voluntad de las propias instituciones, ${ }^{10}$ como ilustra el siguiente gráfico:

$8 \quad$ Se incluye en está cifra la Universidad La República, institución que luego de no ser acreditada en 2006, se encuentra en proceso de cierre, sin embargo, por no contar con datos institucionales actualizados, fue omitida de todos los análisis de este trabajo.

9 Corresponde a la Universidad Gabriela Mistral, que contando con la autonomía desde 1993 no se había sometido a este proceso.

10 Corresponde a 5 universidades, un primer grupo, que está en fase de licenciamiento, no habiendo alcanzado aún su autonomía (lo que las limita a iniciar un proceso de acreditación según lo establecido en la Ley $\mathrm{N}^{\circ}$ 20.129), siendo estas la Universidad Regional San Marcos, Chileno-Británica de Cultura y Miguel de Cervantes (recientemente autónoma); por otra parte, la Universidad Marítima que mediante un convenio transfirió su matrícula el año 2007 a la Universidad Andrés Bello, siendo recientemente autorizada por el Ministerio de Educación para funcionar como Universidad Los Leones como heredera legal de la institución, y finalmente, la Universidad de Aconcagua, quién siendo autónoma desde 2006, no se ha sometido a proceso de acreditación. 
Gráfico 1. Estado del proceso de acreditación institucional al año 2010

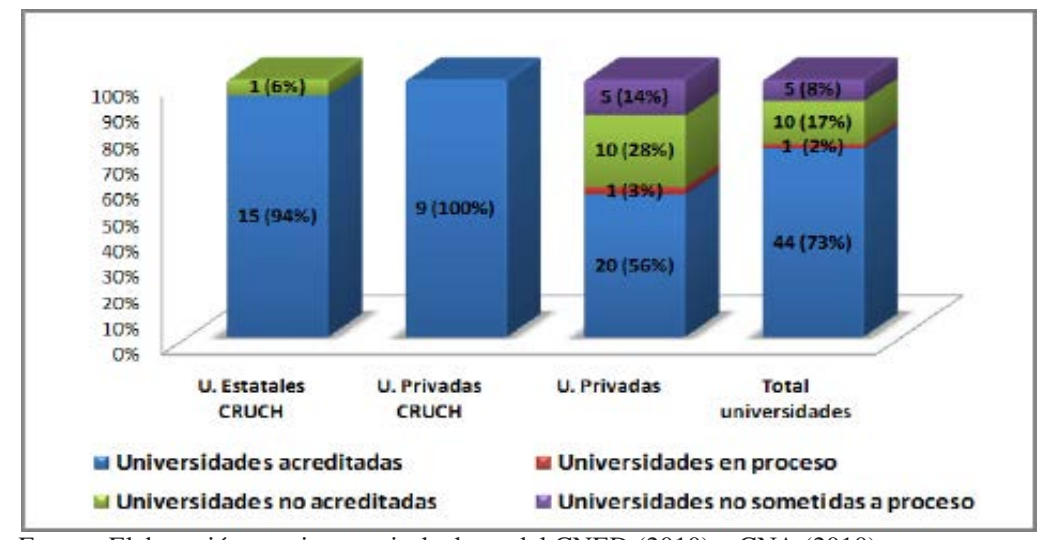

Fuente: Elaboración propia a partir de datos del CNED (2010) y CNA (2010)

Al mismo tiempo, se observa que las instituciones pertenecientes al Consejo de Rectores se encuentran en su mayoría acreditadas ( 24 de las 25 universidades), lo que representa un $96 \%$ de las instituciones que conforman está agrupación (100\% de las universidades privadas del $\mathrm{CRUCH}^{11}$ y un $94 \%$ de las universidades estatales), mientras que entre las universidades privadas (post reforma de 1981) un 57,1\% han logrado la acreditación institucional, mientras que un $28 \%$ de estas instituciones no han sido acreditadas, y un $14 \%$ no se ha sometido a proceso.

Considerando los antecedentes iniciales, los análisis realizados posteriormente incluyen a 53 sometidas a procesos de acreditación, de las 60 universidades existentes, excluyéndose aquellas no sometidas a proceso ( 5 instituciones), una universidad que se encuentra en proceso de acreditación y otra no acreditada sobre la cual no existe información actualizada (U. de La República).

El Anexo 2, elaborado con cifras 2010, muestra diferencias relevantes entre universidades acreditadas institucionalmente, es así como las universidades privadas tradicionales presentan el mayor promedio de años de acreditación (5,56 años), mientras que las estatales cuentan con un promedio de 4 años y las privadas 3,75 años. Del mismo modo, y dando cuenta de su complejidad organizacional, son las universidades privadas tradicionales las que tienen un mayor número de áreas acreditadas, 4 en promedio, las estatales con 3,27 áreas y las privadas con 2,35 áreas de acreditación, lo que sitúa levemente por encima de las 2 áreas de acreditación obligatorias (gestión institucional y docencia conducente a título), en ambos casos al agrupar por universidades pertenecientes al CRUCH y privadas, existe diferencia estadísticamente significativa (ver anexo 3).

En cuanto a la acreditación de áreas opcionales (investigación, postgrado y vinculación con el medio), el siguiente gráfico muestra el número de universidades y las áreas opcionales que han acreditado:

11 Corresponde al grupo de universidades que pese a ser privadas, recibe aporte financiero del Estado a través de sus instrumentos (aporte fiscal directo y aporte fiscal indirecto). 
Gráfico 2. Acreditación de áreas opcionales según tipo de universidad

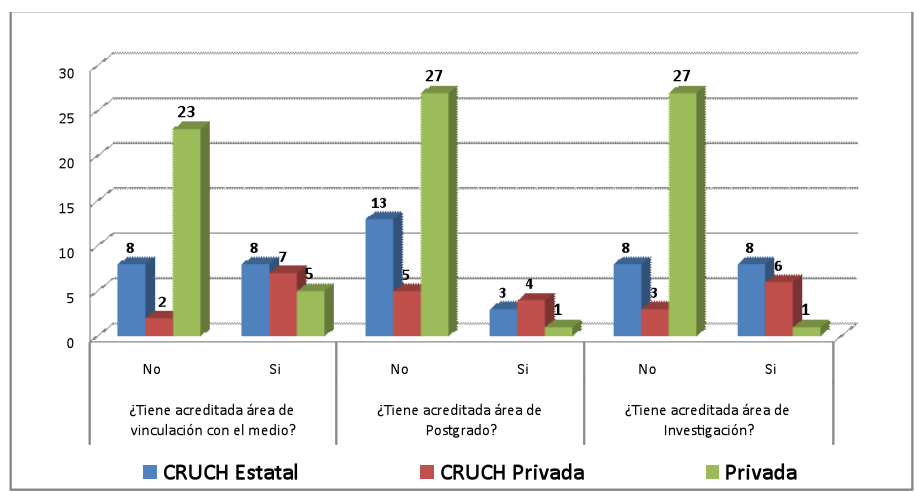

Fuente: Elaboración propia según datos CNA (2010)

Como se puede observar, existen diferencias relevantes entre las universidades pertenecientes al Consejo de Rectores (así como entre las universidades privadas tradicionales y estatales que lo componen), las que alcanzan un mayor grado de acreditación en todas las áreas opcionales que las universidades privadas, lo que se traduce en una asociación estadísticamente significativa para las áreas de investigación y vinculación con el medio (ver resultados de prueba chi-cuadrado en anexos 5 y 6 ), lo que se explica por los siguientes datos:

- En el área de investigación, un 50\% de las universidades estatales y 67\% de las privadas tradicionales han acreditado esta área, lo que representa un $60 \%$ de universidades del CRUCH, en relación a un $4 \%$ de las universidades privadas.

- La acreditación del área de postgrado, ha sido alcanzada por un $19 \%$ de universidades estatales y $44 \%$ de universidades privadas tradicionales, representando un $28 \%$ de universidades del CRUCH, en relación a un $4 \%$ de universidades privadas.

- El área de vinculación con el medio, ha sido acreditada por un 50\% de universidades estatales y $78 \%$ de universidades privadas tradicionales, representando un $56 \%$ de universidades del CRUCH, e comparación a un $18 \%$ de universidades privadas.

Otro ámbito donde se observan diferencias relevantes es el estado de acreditación institucional en relación a la ubicación geográfica de la sede principal de la universidad, haciendo la distinción entre universidades de la Región Metropolitana con un 75\% de instituciones acreditadas y universidades de otras Regiones, donde un $92 \%$ se encuentran acreditadas, como muestra la siguiente Tabla 2:

Tabla 2. Estado de Acreditación Institucional según Ubicación Geográfica Sede Principal

\begin{tabular}{|c|c|c|c|c|}
\hline \multirow{2}{*}{\multicolumn{2}{|c|}{ Estatus de acreditación }} & \multicolumn{2}{|c|}{$\begin{array}{c}\text { Tipo de Universidad } \\
\text { Según Ubicación Sede } \\
\text { Principal }\end{array}$} & \\
\hline & & Metropolitana & $\begin{array}{c}\text { Otras } \\
\text { Regiones }\end{array}$ & \\
\hline \multirow[b]{2}{*}{ No acreditada } & Recuento & 7 & 2 & 9 \\
\hline & $\begin{array}{l}\text { \% dentro de Tipo de Universidad } \\
\text { Según Ubicación Sede Principal }\end{array}$ & $25 \%$ & $8 \%$ & $17 \%$ \\
\hline
\end{tabular}




\begin{tabular}{|c|c|c|c|c|}
\hline \multirow{5}{*}{ Acreditada } & Recuento & 21 & 23 & 44 \\
\cline { 2 - 5 } & $\begin{array}{c}\text { \% dentro de Tipo de Universidad } \\
\text { Según Ubicación Sede Principal }\end{array}$ & $75 \%$ & $92 \%$ & $83 \%$ \\
\hline \multirow{3}{*}{ Total } & $\begin{array}{c}\text { Recuento } \\
\text { Según Ubicación Sede Principal }\end{array}$ & $100,0 \%$ & $100,0 \%$ & $100,0 \%$ \\
\cline { 2 - 5 } & Sentro de Tipo de Universidad & 28 & 25 & 53 \\
\hline
\end{tabular}

Fuente: Datos Comisión Nacional de Acreditación, CNA (2010)

Si bien el estatus de acreditación institucional no evidencia un grado de asociación estadística con la ubicación geográfica de la universidad, en las áreas opcionales de acreditación las universidades regionales muestran un porcentaje superior de instituciones acreditadas en el área de vinculación con el medio, contando con un $65 \%$ entre este subgrupo, mientras que entre las universidades metropolitanas solo un $35 \%$ se encuentra acreditada, lo que evidencia una asociación estadísticamente significativa (ver Anexo 7).

La complejidad del proceso de acreditación institucional ha requerido de la instalación de dispositivos que contribuyan a orientar y ejecutar las acciones internas y externas conducentes a enfrentar las distintas fases que demanda este proceso. Para las universidades, un indicador de éxito del proceso de acreditación se asocia normalmente a la cantidad de años de acreditación obtenidos, y a las áreas opcionales que se logran acreditar. En la Tabla 3 se puede observar que el número de años de acreditación que obtiene una universidad posee un importante nivel de correlación con distintas variables institucionales (ver Anexo 3).

Tabla 3. Coeficientes de Correlación asociados a $\mathrm{N}^{\circ}$ de años de Acreditación Institucional

\begin{tabular}{|c|c|}
\hline Indicadores & $\begin{array}{c}\text { Coeficiente de Correlación } \\
\text { (R de Pearson)* }\end{array}$ \\
\hline Número de área acreditadas & 0,911 \\
\hline Número de docentes full time & 0,698 \\
\hline Número de docentes con postgrado & 0,637 \\
\hline $\begin{array}{c}\text { Número de académicos full time con grado de } \\
\text { doctor }\end{array}$ & 0,607 \\
\hline $\begin{array}{c}\text { Número de proyectos FONDECYT adjudicados } \\
\text { en los últimos 3 años }\end{array}$ & 0,509 \\
\hline Número de programas de doctorado acreditados & 0,505 \\
\hline Número de programas de magíster acreditados & 0,475 \\
\hline Número de volúmenes (textos) por alumno & 0,374 \\
\hline Número de laboratorios & 0,371 \\
\hline
\end{tabular}

(*) Correlación a un nivel de confianza de $99 \%$ 


\section{SITUACIÓN DE LA ACREDITACIÓN DE PROGRAMAS DE PREGRADO}

Conjuntamente con el significativo crecimiento del número de instituciones de educación superior, se produce un incremento relevante en la cobertura en este nivel educativo, pasando de un 7,43\% en 1980 a 14,4\% en 1990, 27,7\% en 2000 (Bernasconi y Rojas, 2003 ) y una estimación por sobre el $40 \%$ en 2010 . Actualmente existe una matrícula total de más de 900 mil estudiantes en educación superior (CNED, 2010a), con una matrícula universitaria que representa un $62 \%$ de la matrícula total. El siguiente gráfico muestra el crecimiento de la matrícula universitaria por tipo de institución:

Gráfico 3. Crecimiento de la matrícula total por tipo de universidad

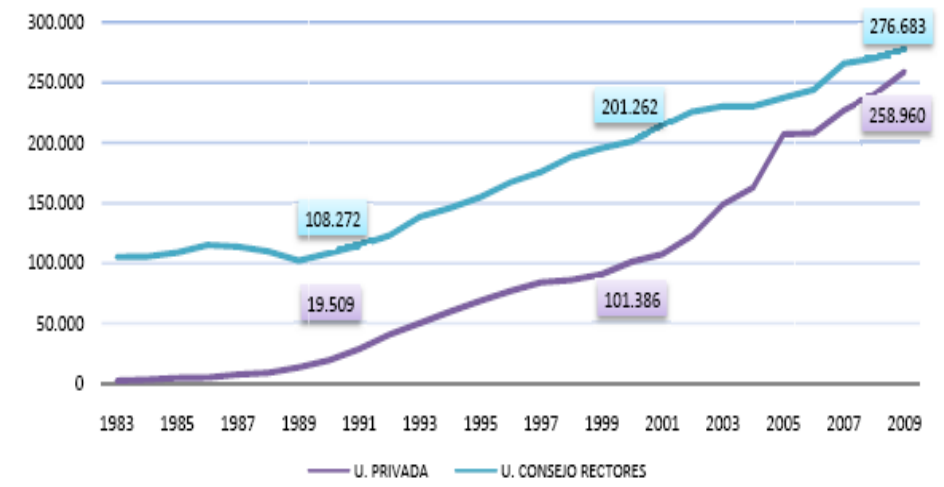

Fuente: Mineduc (2010)

El proceso de acreditación de pregrado ha crecido explosivamente a partir de la obligatoriedad en la acreditación de las carreras del área de pedagogía y medicina, establecido en el artículo $\mathrm{N}^{\circ} 27$ de la Ley $\mathrm{N}^{\circ} 20.129$ del año 2006, lo que ha sido factible también producto de la creación de agencias privadas de acreditación, LAS que nacen a partir de la aprobación de dicha normativa.

Las carreras universitarias acreditadas corresponden a un 34\% del total de programas vigentes en 2010 , encontrándose un $7 \%$ en proceso de acreditación, un $2 \%$ no acreditada y un $57 \%$ que no se ha sometido a proceso, como muestra la siguiente tabla:

Tabla 4. Situación de Acreditación de Carreras de Pregrado por Tipo de Institución

\begin{tabular}{|c|c|c|c|c|c|c|c|c|c|c|c|}
\hline \multirow[b]{2}{*}{$\begin{array}{c}\text { Tipo de } \\
\text { institución }\end{array}$} & \multirow[b]{2}{*}{$\begin{array}{l}\text { Acreditación } \\
\text { Institucional }\end{array}$} & \multirow[b]{2}{*}{$\begin{array}{c}\mathrm{N}^{\circ} \\
\text { instituciones }\end{array}$} & \multicolumn{9}{|c|}{ Carreras } \\
\hline & & & Acreditadas & $\%$ & \begin{tabular}{l|} 
En \\
Proceso
\end{tabular} & $\%$ & $\begin{array}{l}\text { No } \\
\text { Acreditadas }\end{array}$ & $\%$ & \begin{tabular}{|l|} 
No \\
sometidas
\end{tabular} & $\%$ & Total \\
\hline \multirow{2}{*}{$\begin{array}{l}\text { Consejo } \\
\text { de } \\
\text { Rectores } \\
\end{array}$} & Acreditadas & 23 & 400 & $31 \%$ & 100 & $8 \%$ & 27 & $2 \%$ & 777 & $60 \%$ & 1304 \\
\hline & en Proceso & 2 & 7 & $10 \%$ & 3 & $4 \%$ & 3 & $4 \%$ & 58 & $82 \%$ & 71 \\
\hline \multirow[b]{2}{*}{ Privadas } & Acreditadas & 23 & 306 & $22 \%$ & 100 & $7 \%$ & 30 & $2 \%$ & 946 & $68 \%$ & 1382 \\
\hline & en Proceso & 5 & 676 & $50 \%$ & 105 & $8 \%$ & 22 & $2 \%$ & 554 & $41 \%$ & 1357 \\
\hline Totales & & 53 & 1389 & $34 \%$ & 308 & $7 \%$ & 82 & $2 \%$ & 2335 & $57 \%$ & 4114 \\
\hline
\end{tabular}

Fuente: Adaptado a partir de CNED (2010b) 
Las universidades del Consejo de Rectores tienen en promedio 16,3 carreras acreditadas (14,3 las universidades estatales y 19,7 en privadas tradicionales), mientras que las universidades privadas llegan a 7,25 carreras, lo que representa una diferencia estadísticamente significativa. ${ }^{12} \mathrm{El}$ siguiente gráfico muestra el promedio de carreras acreditadas por tipo de institución, donde las universidades privadas del Consejo de Rectores tienen un promedio de 19,67 carreras acreditadas, siguiendo las estatales y finalmente las privadas.

Gráfico 4. Promedio de carreras de pregrado acreditadas por tipo de universidad

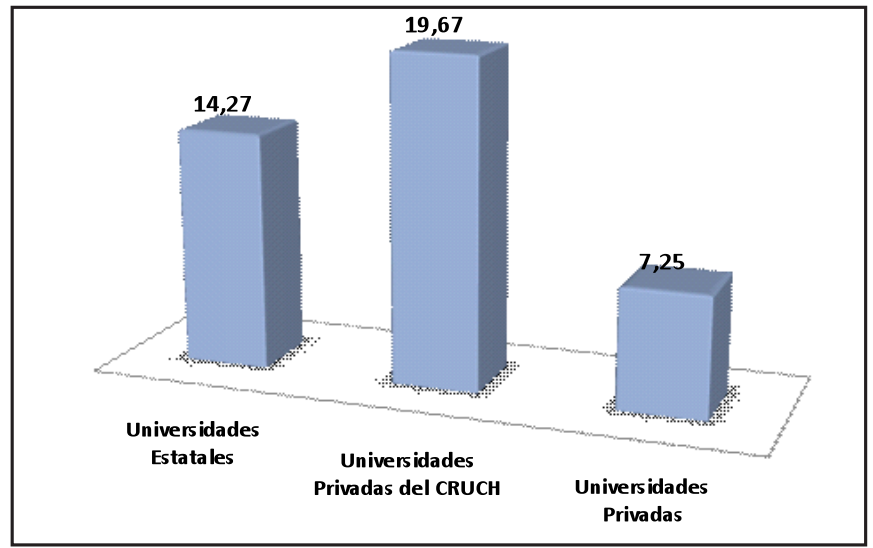

Fuente: Elaboración propia a partir de datos de CNA(2010).

Adicionalmente, el promedio de años de acreditación de las carreras de las universidades Privadas del CRUCH es de 4,4 años, en las universidades estatales llega a 3,6 años, y en las universidades privadas es de 3,2 años, lo que representa en una diferencia estadísticamente significativa entre universidades del CRUCH y privadas (Anexo 3) . Al mismo tiempo, el número de carreras acreditadas que posee una universidad tiene un importante nivel de correlación con distintas variables institucionales (ver Anexo 3), como muestra la siguiente tabla:

Tabla 5. Coeficientes de Correlación asociados a $\mathrm{N}^{\circ}$ de Carreras Acreditadas

\begin{tabular}{|c|c|}
\hline Indicadores & $\begin{array}{c}\text { Coeficiente de Correlación } \\
\text { (R de Pearson)* }\end{array}$ \\
\hline Número de académicos full time con grado de doctor & 0,756 \\
\hline Número de docentes con postgrado & 0,744 \\
\hline Número de programas de doctorado acreditados & 0,717 \\
\hline Número de docentes full time & 0,697 \\
\hline $\begin{array}{c}\text { Número de proyectos FONDECYT adjudicados en } \\
\text { los últimos 3 años }\end{array}$ & 0,690 \\
\hline
\end{tabular}

12 Resultado del la prueba T-Student, con un nivel del confianza del 95\% (ver Anexo 4). 


\begin{tabular}{|c|l|}
\hline Número de programas de magíster acreditados & 0,679 \\
\hline Promedio años de acreditación carreras & 0,598 \\
\hline Número de laboratorios & 0,521 \\
\hline Metros cuadrados de laboratorio & 0,511 \\
\hline Número de área acreditadas & 0,497 \\
\hline Número de área acreditadas & 0,497 \\
\hline
\end{tabular}

(*) Correlación a un nivel de confianza de $99 \%$

\section{SITUACIÓN DE LA ACREDITACIÓN DE PROGRAMAS DE POSTGRADO}

Las cifras de 2010 que se presentan en la Tabla 6 muestran que las universidades del Consejo de Rectores han acreditado un 33,7\% de sus programas de magíster y un $92,4 \%$ de los programas de doctorado, llegando a un promedio por institución de 6,64 programas de magíster y 4,48 programas de doctorados, mientras que las universidades privadas han acreditado un $8,8 \%$ de sus magíster y $54,5 \%$ de programas de doctorado, alcanzando un promedio por universidad de 0,18 programas de magíster y 0,21 programas de doctorado, lo que representa una diferencia estadísticamente significativa. ${ }^{13}$

Tabla 6. Situación de Acreditación de Programas de Postgrado por Tipo de Institución

\begin{tabular}{|c|c|c|c|c|c|c|c|}
\hline $\begin{array}{c}\text { Tipo de } \\
\text { Institución }\end{array}$ & $\begin{array}{c}\text { Acreditación } \\
\text { Institucional }\end{array}$ & $\begin{array}{c}\mathrm{N}^{\circ} \text { Programas } \\
\text { de Magíster }\end{array}$ & $\begin{array}{c}\mathrm{N}^{\circ} \text { Programas } \\
\text { de Magíster } \\
\text { Acreditados }\end{array}$ & $\%$ & $\begin{array}{c}\mathrm{N}^{\circ} \\
\text { Programas } \\
\text { de } \\
\text { doctorados }\end{array}$ & $\begin{array}{c}\mathrm{N}^{\circ} \\
\text { Programas } \\
\text { de } \\
\text { doctorado } \\
\text { acreditados }\end{array}$ & $\%$ \\
\hline $\begin{array}{c}\text { Consejo de } \\
\text { Rectores }\end{array}$ & Acreditada & 493 & 166 & $33,7 \%$ & 132 & 122 & $92,4 \%$ \\
\cline { 2 - 9 } & No acreditada & 10 & 0 & $0,0 \%$ & 6 & 0 & $0,0 \%$ \\
\hline \multirow{2}{*}{ Privadas } & Acreditada & 239 & 21 & $8,8 \%$ & 11 & 6 & $54,5 \%$ \\
\cline { 2 - 9 } & No acreditada & 43 & 0 & $0,0 \%$ & 3 & 0 & $0,0 \%$ \\
\hline \multicolumn{2}{|r|}{ Totales } & 785 & 187 & $23,8 \%$ & 152 & 128 & $84,2 \%$ \\
\hline
\end{tabular}

Fuente: Elaboración Propia a partir de CNA (2010), CNED (2010b), CNED (2010a).

El siguiente gráfico, muestra el promedio de programas de postgrado acreditados por tipo de institución, donde las universidades privadas del CRUCH tienen un promedio de 8,78 magister y 8 programas de doctorados acreditados, siguiendo las estatales con 5,44 magíster y 3,13 doctorados, y finalmente las privadas con 0,18 magíster y 0,21 doctorados.

13 Resultado del la prueba T-Student, con un nivel del confianza del 95\% (ver Anexo 4). 
Gráfico 5. Promedio de programas de postgrados acreditados por tipo de universidad

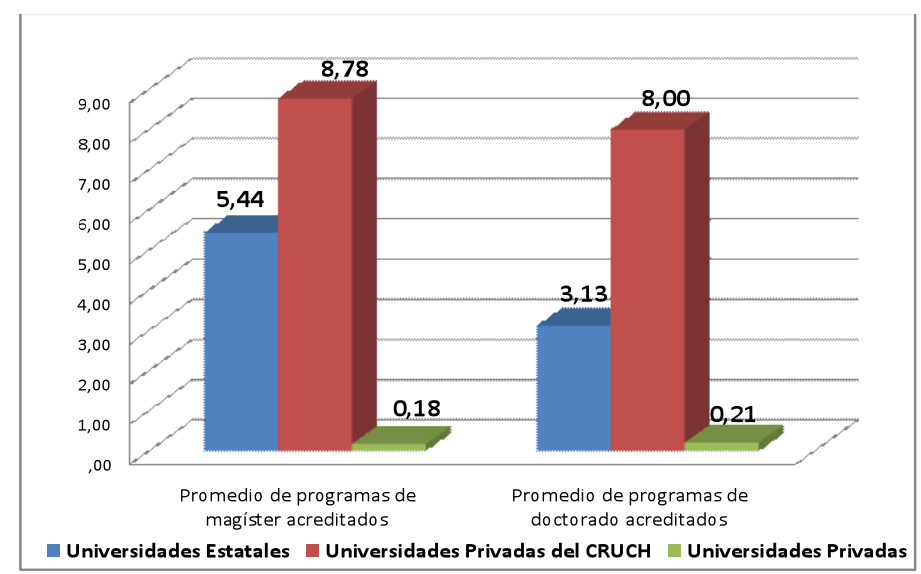

Fuente: Elaboración propia a partir de datos de CNA (2010).

Adicionalmente, el promedio de años de acreditación de los programas de magíster llega a 3,88 para las universidades privadas del CRUCH, 3,47 años para las estatales y 3,38 años para las universidades privadas. A nivel de doctorado, el promedio de años de acreditación es de 4,58 para las universidades privadas del $\mathrm{CRUCH}, 3,84$ para las universidades estatales y 3,3 años para las privadas. La siguiente tabla muestra el alto grado de correlación existente entre el número de programas de doctorados acreditados por universidad con las variables que se especifican:

Tabla 7. Coeficientes de Correlación asociados a $N^{\circ}$ de Programas de Doctorado Acreditados

\begin{tabular}{|c|c|}
\hline $\begin{array}{c}\text { Indicadores } \\
\text { Número de proyectos FONDECYT adjudicados en } \\
\text { los últimos 3 años }\end{array}$ & Coeficiente de Correlación \\
\hline Número de magíster acreditados & 0,979 \\
\hline Número de académicos full time con grado de doctor & 0,971 \\
\hline Número de docentes con postgrado & 0,952 \\
\hline Número de docentes full time & 0,913 \\
\hline Número de laboratorios & 0,886 \\
\hline Número de carreras acreditadas & 0,735 \\
\hline Número de textos por alumnos & 0,717 \\
\hline Metros cuadrados de laboratorio & 0,703 \\
\hline Promedio de años de acreditación de carreras & 0,671 \\
\hline Número de área acreditadas & 0,625 \\
\hline Número de años de acreditación institucional & 0,546 \\
\hline
\end{tabular}

14 Se utiliza el concepto "meritocracia", entendido como el mérito asociado a la acumulación de un conjunto de recursos institucionales o stock de capacidades, que hace a una institución merecedora de un logro determinado. 
Una situación equivalente ocurre con las variables números de programas de magíster acreditados, con el mismo conjunto de variables analizados (para más detalle ver Anexo 3 ). Estos datos no hacen más que confirmar el carácter meritocrático de los procesos de acreditación de programas de pregrado y postgrado, y la relevancia que tienen indicadores convencionales de desempeño universitario que reflejan el soporte institucional con que cuentan universidades para el desarrollo de sus actividades académicas y de investigación.

Por otra parte, como fue señalado anteriormente, el número de carreras de pregrado acreditadas muestra una alta correlación con las variables número de programas de magíster acreditados $(\mathrm{R}=0,679)$ y número de programas de doctorado acreditados $(\mathrm{R}=0,717)$, lo que estaría reflejando una sinergia entre los procesos de acreditación de programas de pre y postgrado, producto de la institucionalización de unidades organizacionales, políticas y procesos orientados hacia la instalación de una cultura de evaluación.

\section{CONCLUSIONES}

La acreditación de las universidades en Chile se ha instalado paulatinamente, alcanzando un nivel de desarrollo relevante desde 2003, para llegar actualmente (2010) a contar con un $73 \%$ de instituciones acreditadas, y un $17 \%$ que no lo logró, un $2 \%$ que está en proceso, y un $8 \%$ de las universidades no han sido sometidas a esta instancia, por no cumplir con los requisitos legales para ello.

El sistema muestra cifras que marcan diferencias relevantes en la cantidad de años de acreditación, el número de áreas opcionales que logran acreditar las universidades (investigación, postgrado o vinculación con el medio), así como el número y años de acreditación de carreras y programas de postgrado, según sean universidades privadas o tradicionales (universidades del Consejo de Rectores), e inclusive entre las mismas universidades del $\mathrm{CRUCH}$ (estatales y privadas tradicionales). Lo descrito señala la existencia de una diversidad cualitativa importante en la oferta educativa por parte de las universidades chilenas.

El éxito de un proceso de acreditación institucional normalmente se asocia a la cantidad de años de acreditación que se otorga a una universidad (así como también el número de áreas opcionales que pueda acreditar), encontrándose una fuerte asociación con variables sinérgicas, como es: el número de programas postgrado acreditados (magíster y doctorado), indicadores académicos convencionales $\left(\mathrm{n}^{\circ}\right.$ académicos full time, $\mathrm{n}^{\circ}$ de docentes con postgrado, número de académicos con grado de doctor, $\mathrm{n}^{\circ}$ de proyectos Fondecyt adjudicados), así como otros indicadores de soporte institucional.

Una situación equivalente ocurre con la acreditación de programas de pregrado y postgrado, lo que muestra que la acreditación tiende a arrojar resultados positivos para aquellas universidades donde se encuentran instalados recursos, capacidades y políticas institucionales en la materia, consecuentemente, se crean procesos sinérgicos en todos los ámbitos de la acreditación (círculo virtuoso), transformándose en un proceso de carácter meritocrático, donde variables convencionales de dotación de capital humano avanzado y de productividad académica, contribuyen favorablemente a la obtención de un mejor desempeño institucional.

Los resultados de los procesos de acreditación (en todos los ámbitos), evidencian un alto grado de asociación con el grado de desarrollo institucional de las universidades, 
donde las universidades privadas tradicionales (pertenecientes al CRUCH) cuentan con una mejor performance. Estos resultados se explicarían porque este subgrupo de universidades está compuesto por 6 universidades (de 9 instituciones) con una larga trayectoria académica (de al menos 50 años), en comparación con las universidades estatales, donde sólo 2 de las 16 instituciones tienen una trayectoria equivalente, mientras que las restantes 14 universidades estatales nacen a partir de la reforma de 1981, al igual que las universidades privadas no pertenecientes al CRUCH.

Lo señalado anteriormente plantea la necesidad de buscar nuevos patrones de comparación que, de alguna manera, den cuenta de la diversidad del sistema universitario donde conviven universidades con distinto grado de complejidad. Algunas abarcan todos los ámbitos académicos convencionales, otras que se focalizan en ámbitos específicos del quehacer universitario (como la extensión, el postgrado y/o la investigación), o están concentradas preferentemente en la docencia de pregrado, en función de un proceso selectivo de estrategias conducentes a la consolidación de un proyecto educativo específico. De la misma forma, existen diversos factores, como el anclaje territorial, que han influido en los procesos de desarrollo institucional debido a limitaciones en la accesibilidad a recursos de toda índole (humanos, tecnológicos, financieros, entre otros) y el acceso a sub mercados de menor escala.

El alto grado de vinculación entre indicadores universitarios tradicionales e indicadores de acreditación, revela la necesidad de contar con sistemas de información públicos más complejos que permitan contar con antecedentes institucionales relevantes, de manera de disponer de estándares de comparación (benchmarking) conducentes al fortalecimiento de los procesos evaluativos, en beneficio de la mejora continua de las universidades y del sistema de educación superior chileno.

\section{ANEXOS}

Anexo 1. Evolución del Número de Instituciones de Educación Superior en Chile

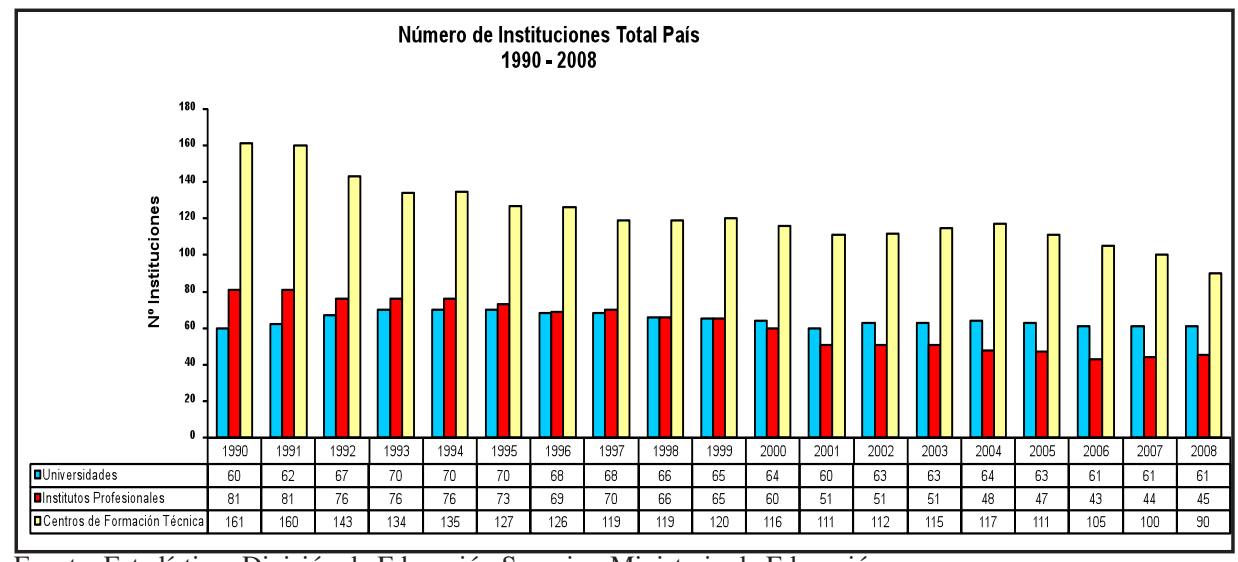

Fuente: Estadísticas División de Educación Superior, Ministerio de Educación. 
Anexo 2. Estadísticos de indicadores de instituciones acreditadas según dependencia

\begin{tabular}{|c|c|c|c|c|c|}
\hline Indicador & Tipo de Universidad & Media & Desviación típica & Mínimo & Máximo \\
\hline \multirow{4}{*}{$\begin{array}{l}\mathrm{N}^{\circ} \text { de años de } \\
\text { acreditación } \\
\text { institucional }\end{array}$} & Estatal & 4,00 & 1,56 & 1,00 & 7,00 \\
\hline & Privada $\mathrm{CRUCH}$ & 5,56 &, 88 & 4,00 & 7,00 \\
\hline & Privada Post Reforma 1981 & 3,75 & 1,21 & 2,00 & 6,00 \\
\hline & Total & 4,20 & 1,44 & 1,00 & 7,00 \\
\hline \multirow{4}{*}{$\begin{array}{l}\text { Número de áreas } \\
\text { acreditadas }\end{array}$} & Estatal & 3,27 & 1,16 & 2,00 & 5,00 \\
\hline & Privada $\mathrm{CRUCH}$ & 4,00 & 1,00 & 3,00 & 5,00 \\
\hline & Privada Post Reforma 1981 & 2,35 & 67 & 2,00 & 4,00 \\
\hline & Total & 3,00 & 1,12 & 2,00 & 5,00 \\
\hline \multirow{4}{*}{$\begin{array}{l}\text { Número de } \\
\text { carreras } \\
\text { acreditadas }\end{array}$} & Estatal & 14,27 & 9,37 & 3,00 & 37,00 \\
\hline & Privada $\mathrm{CRUCH}$ & 19,67 & 12,25 & 3,00 & 36,00 \\
\hline & Privada Post Reforma 1981 & 7,25 & 4,06 & 1,00 & 14,00 \\
\hline & Total & 11,42 & 8,96 & 1,00 & 37,00 \\
\hline \multirow{4}{*}{$\begin{array}{l}\text { Promedio años de } \\
\text { acreditación de } \\
\text { carreras }\end{array}$} & Estatal & 3,58 &, 91 & 2,38 & 5,51 \\
\hline & Privada $\mathrm{CRUCH}$ & 4,37 & 1,11 & 3,00 & 6,70 \\
\hline & Privada Post Reforma 1981 & 3,23 &, 89 & 1,00 & 4,50 \\
\hline & Total & 3,53 & 1,01 & 1,00 & 6,70 \\
\hline \multirow{4}{*}{$\begin{array}{l}\mathrm{N}^{\circ} \text { de programas } \\
\text { de magister } \\
\text { acreditados }\end{array}$} & Estatal & $\overline{5,44}$ & 12,93 &, 00 & 53,00 \\
\hline & Privada $\mathrm{CRUCH}$ & 8,78 & 11,56 &, 00 & 31,00 \\
\hline & Privada Post Reforma 1981 &, 18 &, 77 &, 00 & 4,00 \\
\hline & Total & 3,23 & 8,99 &, 00 & 53,00 \\
\hline \multirow{4}{*}{$\begin{array}{l}\text { Promedio años de } \\
\text { acreditación } \\
\text { programas de } \\
\text { magíster }\end{array}$} & Estatal & 3,47 &, 95 & 2,00 & 5,50 \\
\hline & Privada $\mathrm{CRUCH}$ & 3,88 &, 98 & 2,00 & 5,00 \\
\hline & Privada Post Reforma 1981 & 3,38 & 1,94 & 2,00 & 4,75 \\
\hline & Total & 3,60 & 1,01 & 2,00 & 5,50 \\
\hline \multirow{4}{*}{$\begin{array}{l}\mathrm{N}^{\circ} \text { de programas } \\
\text { de doctorado } \\
\text { acreditados }\end{array}$} & Estatal & 3,13 & 8,44 &, 00 & 34,00 \\
\hline & Privada $\mathrm{CRUCH}$ & 8,00 & 10,09 &, 00 & 29,00 \\
\hline & Privada Post Reforma 1981 &, 21 &, 63 &, 00 & 3,00 \\
\hline & Total & 2,42 & 6,67 &, 00 & 34,00 \\
\hline \multirow{4}{*}{$\begin{array}{l}\text { Promedio años de } \\
\text { acreditación } \\
\text { programas de } \\
\text { doctorado }\end{array}$} & Estatal & 3,84 & ,72 & 3,00 & 4,71 \\
\hline & Privada $\mathrm{CRUCH}$ & 4,58 &, 82 & 3,25 & 5,75 \\
\hline & Privada Post Reforma 1981 & 3,33 & 1,41 & 2,00 & 5,33 \\
\hline & Total & 3,98 & 1,02 & 2,00 & 5,75 \\
\hline
\end{tabular}


Anexo 3. Matriz de correlación de indicadores institucionales

\begin{tabular}{|c|c|c|c|c|c|c|c|c|c|c|c|c|c|c|c|c|c|}
\hline & & 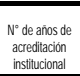 & $\begin{array}{l}\text { Nimerer de } \\
\text { areas } \\
\text { acedetiadas }\end{array}$ & $\begin{array}{l}\text { Nimertode } \\
\text { carras } \\
\text { acceditadas }\end{array}$ & 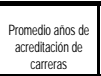 & $\begin{array}{c}\text { Node } \\
\text { programas de } \\
\text { magister } \\
\text { acceditados }\end{array}$ & 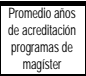 & $\begin{array}{c}N^{v} \text { de } \\
\text { programas de } \\
\text { doctorado } \\
\text { acreditados }\end{array}$ & 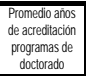 & \begin{tabular}{|c|}
$N^{\circ}$ \\
ocoenes \\
tull time
\end{tabular} & \begin{tabular}{|c|}
$N^{\circ}$ \\
onctores \\
tull ine
\end{tabular} & $\left|\begin{array}{c}N^{N} \text { docentes } \\
\text { con } \\
\text { posggarado }\end{array}\right|$ & 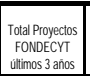 & \begin{tabular}{|c|} 
Mis.2.2de \\
bibioficea \\
por \\
alumno \\
\end{tabular} & 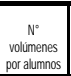 & \begin{tabular}{|l|} 
Mis.2de \\
labocatoloios
\end{tabular} & $\begin{array}{l}\text { No de } \\
\text { laboratoroios }\end{array}$ \\
\hline $\begin{array}{l}N^{\circ} \text { de a año de } \\
\text { acreditación } \\
\text { institucional }\end{array}$ & $\begin{array}{l}\text { Conelación de Pearson } \\
\text { Sig. (bialteral) } \\
\mathrm{N}\end{array}$ & 1 & \begin{tabular}{r|}
, $911 "$ \\
.000 \\
53 \\
\end{tabular} & \begin{tabular}{|r|}
, 352 \\
, 011 \\
52 \\
\end{tabular} & \begin{tabular}{r|}
, 346 \\
, 012 \\
52 \\
\end{tabular} & $\begin{array}{r}., 45^{\prime \prime} \\
.000 \\
53\end{array}$ & \begin{tabular}{|l|}
, 375 \\
, 103 \\
20 \\
\end{tabular} & \begin{tabular}{r|}
, $50{ }^{\prime \prime}$ \\
.000 \\
53
\end{tabular} & \begin{tabular}{|l|}
.386 \\
.126 \\
17
\end{tabular} & \begin{tabular}{r|}
, 584 \\
.000 \\
52 \\
\end{tabular} & \begin{tabular}{r|}
, $607 "$ \\
, 000 \\
52 \\
\end{tabular} & \begin{tabular}{r|}
, $637 "$ \\
.000 \\
52
\end{tabular} & \begin{tabular}{r|}
, $500^{\prime \prime}$ \\
, 000 \\
53
\end{tabular} & \begin{tabular}{r|}
, 347 \\
.011 \\
53 \\
\end{tabular} & \begin{tabular}{r|}
, $374^{\prime \prime}$ \\
.066 \\
53 \\
5
\end{tabular} & \begin{tabular}{r|}
, $306^{\circ}$ \\
, 026 \\
53 \\
\end{tabular} & $\begin{array}{r}, 371^{\prime \prime} \\
, 006 \\
53\end{array}$ \\
\hline $\begin{array}{l}\text { Númerero de } \\
\text { àreas } \\
\text { acteditiadas }\end{array}$ & $\begin{array}{l}\text { Correación de Pearson } \\
\text { Sig., (biatera) } \\
\text { N }\end{array}$ & $\begin{array}{l}.911^{\prime \prime} \\
.000 \\
53\end{array}$ & 1 & $\begin{array}{l}.497 " \\
.000 \\
52 \\
\end{array}$ & $\begin{array}{r}.444^{\prime \prime} \\
.001 \\
52 \\
\end{array}$ & $\begin{array}{l}.518^{\prime \prime} \\
.000 \\
53\end{array}$ & $\begin{array}{r}.339 \\
.144 \\
20 \\
\end{array}$ & $\begin{array}{c}.546 " \\
.000 \\
53\end{array}$ & $\begin{array}{r}.443 \\
.075 \\
17\end{array}$ & $\begin{array}{r}.630 " \\
.000 \\
52 \\
\end{array}$ & $\begin{array}{r}.668 " \\
.000 \\
52 \\
52\end{array}$ & \begin{tabular}{|l|}
$.697 "$ \\
.000 \\
52 \\
\end{tabular} & $\begin{array}{r}5.543^{*} \\
.000 \\
53 \\
\end{array}$ & $\begin{array}{r}.294^{\prime} \\
.033 \\
53 \\
\end{array}$ & $\begin{array}{r}.356 " \\
.009 \\
53 \\
\end{array}$ & $\begin{array}{r}.319^{\circ} \\
.020 \\
53 \\
\end{array}$ & $\begin{array}{l}.363^{\prime \prime} \\
.008\end{array}$ \\
\hline 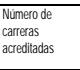 & $\begin{array}{l}\text { Correlacón de Pearson } \\
\text { Sig, (biatera) } \\
\text { N }\end{array}$ & $\begin{array}{r}.352^{2} \\
.011 \\
52\end{array}$ & $\begin{array}{r}.497 " \\
.000 \\
52 \\
\end{array}$ & 1 & $\begin{array}{r}5.598^{\circ} \\
.000 \\
52 \\
\end{array}$ & $\begin{array}{l}, 679^{\prime \prime} \\
.000 \\
52\end{array}$ & \begin{tabular}{r|}
, 254 \\
, 293 \\
19
\end{tabular} & $\begin{aligned} & .717 " \\
& .000 \\
& 52\end{aligned}$ & $\begin{array}{l}., 528^{\prime} \\
.029 \\
17\end{array}$ & $\begin{array}{r}.697 " \\
.000 \\
51 \\
\end{array}$ & $\begin{array}{r}.756 " \\
.000 \\
51 \\
51\end{array}$ & \begin{tabular}{r|}
$.744 "$ \\
.000 \\
51
\end{tabular} & $\begin{array}{r}6,600^{\circ} \\
, 000 \\
52 \\
\end{array}$ & $\begin{array}{l}.064 \\
.654 \\
52 \\
\end{array}$ & \begin{tabular}{r|}
, $304^{\prime}$ \\
, 029 \\
52 \\
\end{tabular} & $\begin{array}{l}., 511^{\prime \prime} \\
.000 \\
52\end{array}$ & $\begin{array}{l}., 521^{\prime \prime} \\
.000 \\
52\end{array}$ \\
\hline 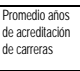 & $\begin{array}{l}\text { Coreacocon de Pearson } \\
\text { Sig., (biaterala } \\
\text { N }\end{array}$ & $\begin{aligned}, 346^{\circ} \\
.012 \\
52\end{aligned}$ & \begin{tabular}{r|r|}
.444 \\
.001 \\
52 \\
5
\end{tabular} & $\begin{array}{l}., 598^{\circ "} \\
.000 \\
52\end{array}$ & 1 & $\begin{array}{l}.583^{\prime \prime} \\
.000 \\
52\end{array}$ & \begin{tabular}{r|}
.232 \\
.340 \\
19
\end{tabular} & $\begin{aligned} & .625 " \\
& .000 \\
& 52\end{aligned}$ & $\begin{array}{l}.597 \\
.011 \\
17 \\
\end{array}$ & $\begin{array}{r}596 " \\
5,000 \\
51 \\
51\end{array}$ & $\begin{array}{r}.658^{\circ} \\
.000 \\
51 \\
51\end{array}$ & \begin{tabular}{|l|}
$.633^{\prime \prime}$ \\
.000 \\
51
\end{tabular} & \begin{tabular}{r|}
, $627^{\circ}$ \\
, 000 \\
52 \\
\end{tabular} & \begin{tabular}{r|}
, 127 \\
.266 \\
52 \\
\end{tabular} & \begin{tabular}{r|}
, 317 \\
.022 \\
52 \\
5
\end{tabular} & $\begin{array}{c}.497 " \\
.000 \\
52\end{array}$ & $\begin{array}{l}.485^{\circ} \\
, 000\end{array}$ \\
\hline 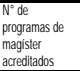 & $\begin{array}{l}\text { Correacion de Pearson } \\
\text { Sig. (bilatera) } \\
\mathrm{N}\end{array}$ & \begin{tabular}{rl|}
$.475^{\prime \prime}$ & \\
.000 \\
53
\end{tabular} & $\begin{array}{r}5.518^{\prime \prime} \\
.000 \\
53\end{array}$ & $\begin{aligned} 679^{\prime \prime} \\
, 000 \\
52\end{aligned}$ & $\begin{array}{r}5.583^{\prime \prime} \\
, 000 \\
52 \\
\end{array}$ & 53 & $\begin{array}{r}.285 \\
.223 \\
20\end{array}$ & $\begin{array}{r}.971 " \\
.000 \\
53\end{array}$ & $\begin{array}{r}, 278 \\
, 279 \\
17\end{array}$ & $\begin{array}{r}8388^{\prime \prime} \\
.000 \\
52 \\
\end{array}$ & $\begin{array}{r}8897 \\
, 000 \\
52 \\
\end{array}$ & $\begin{array}{r}.874^{\prime \prime} \\
.000 \\
52 \\
\end{array}$ & $\begin{array}{l}9666^{\circ} \\
, 000 \\
53\end{array}$ & $\begin{array}{r}.212 \\
.128 \\
53 \\
\end{array}$ & $\begin{array}{l}7,733^{\prime \prime} \\
, 000 \\
53\end{array}$ & $\begin{array}{l}.716 " \\
, 000 \\
53\end{array}$ & $\begin{array}{r}.743^{\prime \prime} \\
.000 \\
53\end{array}$ \\
\hline 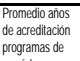 & $\begin{array}{l}\text { Correlación de Pearson } \\
\text { Sig. (bilateral) } \\
N\end{array}$ & $\begin{array}{l}375 \\
, 103\end{array}$ & $\begin{array}{l}, 339 \\
.144 \\
\end{array}$ & $\begin{array}{l}, 254 \\
, 293 \\
, 19\end{array}$ & $\begin{array}{l}.232 \\
, 340 \\
.19\end{array}$ & .225 & 1 & $\begin{array}{l}.344 \\
, 137 \\
, 20\end{array}$ & $\begin{array}{r}-, 293 \\
.332 \\
.13\end{array}$ & $\begin{array}{l}.310 \\
.184 \\
\end{array}$ & $\begin{array}{l}, 358 \\
, 121 \\
20\end{array}$ & \begin{tabular}{l|}
.332 \\
.153 \\
20
\end{tabular} & $\begin{array}{l}, 284 \\
, 226 \\
20\end{array}$ & $\begin{array}{l}.421 \\
.065 \\
20\end{array}$ & $\begin{array}{l}.403 \\
, 078 \\
20\end{array}$ & $\begin{array}{l}309 \\
.186 \\
20\end{array}$ & $\begin{array}{l}.358 \\
, 121\end{array}$ \\
\hline 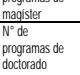 & $\begin{array}{l}\text { N } \\
\text { Conelación de Pearson } \\
\text { Sig., (biateral) } \\
\text { N }\end{array}$ & $\begin{array}{l}20 \\
.505^{\prime \prime} \\
.000 \\
502\end{array}$ & \begin{tabular}{r|}
20 \\
$5.546 "$ \\
, 000 \\
5
\end{tabular} & $\begin{array}{r}199 \\
.717^{\prime \prime} \\
.000 \\
5\end{array}$ & $\begin{array}{r}.625^{\circ} \\
, 000 \\
5.5\end{array}$ & $\begin{array}{l}20 \\
.971^{\prime \prime} \\
.000 \\
52\end{array}$ & , 344 & $\frac{20}{1}$ & $\begin{array}{r}13 \\
, 389 \\
, 123 \\
17\end{array}$ & $\begin{array}{l}20 \\
8.866^{\prime \prime} \\
, 000 \\
55\end{array}$ & $\begin{array}{l}.052 " \\
.000 \\
.52\end{array}$ & \begin{tabular}{r|}
, $913^{\prime \prime}$ \\
.000 \\
50
\end{tabular} & $\begin{array}{r}904 \\
.970^{\circ} \\
, 000 \\
52\end{array}$ & $\begin{array}{ll}, 226 \\
.104 \\
5\end{array}$ & \begin{tabular}{r|}
$.703^{\prime \prime}$ \\
, 000 \\
5.5
\end{tabular} & $\begin{array}{l}.00 \\
.671 " \\
, 000 \\
5\end{array}$ & $\begin{aligned}, 735^{\prime \prime} \\
.000 \\
53\end{aligned}$ \\
\hline acedidiados & $\begin{array}{l}\mathrm{N} \\
\text { Conelacíón de Pearson }\end{array}$ & 53 & 53 & 52 & 52 & 53 & 20 & 53 & 17 & 52 & 52 & 52 & 53 & 53 & 53 & 53 & \\
\hline 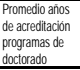 & $\begin{array}{l}\text { Correlación de Peasson } \\
\text { Sig, (biateral) } \\
\mathrm{N}\end{array}$ & $\begin{array}{r}.386 \\
.126 \\
17 \\
\end{array}$ & $\begin{array}{r}., 443 \\
.075 \\
17 \\
\end{array}$ & $\begin{array}{r}.528^{\circ} \\
.029 \\
17 \\
\end{array}$ & $\begin{array}{r}5977 \\
.011 \\
17 \\
\end{array}$ & $\begin{array}{r}278 \\
.279 \\
17 \\
\end{array}$ & $\begin{array}{r}-, 293 \\
.332 \\
13 \\
\end{array}$ & $\begin{array}{r}.389 \\
.123 \\
17 \\
\end{array}$ & 17 & $\begin{array}{r}.532 \\
.028 \\
17 \\
\end{array}$ & $\begin{array}{r}.455 \\
.066 \\
17 \\
\end{array}$ & $\begin{array}{r}.4977 \\
.042 \\
17 \\
\end{array}$ & $\begin{array}{r}.411 \\
, 101 \\
17 \\
\end{array}$ & $\begin{array}{r}-410 \\
, 102 \\
17 \\
\end{array}$ & $\begin{array}{r}-137 \\
.601 \\
17 \\
\end{array}$ & $\begin{array}{r}.308 \\
.229 \\
17 \\
\end{array}$ & $\begin{array}{r}.342 \\
.180 \\
17 \\
\end{array}$ \\
\hline $\begin{array}{l}N^{0} \text { docentes tull } \\
\text { time }\end{array}$ & $\begin{array}{l}\text { Correlacón de Pearson } \\
\text { Sig., (biaterala) } \\
\text { N. }\end{array}$ & $\begin{aligned} &, 584^{\prime \prime} \\
& .000 \\
& 52\end{aligned}$ & $\begin{array}{r}.630^{\circ} \\
.000 \\
52 \\
\end{array}$ & $\begin{array}{l}.697 " \\
.000 \\
51\end{array}$ & $\begin{array}{r}.596 " \\
.000 \\
51 \\
51\end{array}$ & $\begin{array}{l}.838^{\prime \prime} \\
.000 \\
52\end{array}$ & $\begin{array}{l}.310 \\
.184 \\
20\end{array}$ & \begin{tabular}{rl|}
$.886^{\prime \prime}$ & \\
.000 \\
52
\end{tabular} & $\begin{array}{l}.532 \\
.028 \\
.28 \\
17\end{array}$ & 1 & $\begin{array}{r}.953 " \\
.000 \\
52 \\
5\end{array}$ & \begin{tabular}{|l|}
$.976 "$ \\
.000 \\
52
\end{tabular} & $\begin{array}{r}8.873^{4} \\
.000 \\
52 \\
\end{array}$ & $\begin{array}{l}.233 \\
., 996 \\
52 \\
\end{array}$ & $\begin{array}{r}.537^{\prime \prime} \\
.000 \\
52 \\
52\end{array}$ & \begin{tabular}{rl|}
, $602^{\prime \prime}$ & \\
.000 \\
52
\end{tabular} & $\begin{array}{l}.653^{\prime \prime} \\
.000 \\
52\end{array}$ \\
\hline 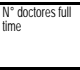 & $\begin{array}{l}\text { Correacón de Pearson } \\
\text { Sig, (bilatera) } \\
\mathrm{N}\end{array}$ & $\begin{array}{r}, 607 " \\
, 000 \\
52 \\
52 \\
\end{array}$ & $\begin{array}{r}, 668^{\prime \prime} \\
, 000 \\
52 \\
\end{array}$ & $\begin{array}{r}.756^{\prime \prime} \\
.000 \\
51 \\
\end{array}$ & $\begin{array}{r}, 658^{\prime \prime} \\
, 000 \\
51 \\
\end{array}$ & $\begin{array}{r}.897^{\prime \prime} \\
.000 \\
52 \\
\end{array}$ & \begin{tabular}{r|}
.358 \\
, 121 \\
20 \\
\end{tabular} & $\begin{aligned} .952 " \\
.000 \\
52 \\
\end{aligned}$ & $\begin{array}{r}.455 \\
.066 \\
17 \\
\end{array}$ & $\begin{array}{r}.953 " \\
.000 \\
52 \\
\end{array}$ & 1 & \begin{tabular}{|l|}
, $980 "$ \\
.000 \\
52 \\
\end{tabular} & $\begin{array}{r}9266^{\prime \prime} \\
, 000 \\
52 \\
\end{array}$ & \begin{tabular}{rl|}
.240 \\
.087 \\
52 \\
\end{tabular} & $\begin{array}{r}, 610^{\circ} \\
, 000 \\
52 \\
\end{array}$ & $\begin{array}{r}, 574^{\prime \prime} \\
, 000 \\
52 \\
\end{array}$ & $\begin{array}{l}, 657^{\prime \prime} \\
, 000 \\
52 \\
\end{array}$ \\
\hline $\begin{array}{l}\mathbb{N}^{*} \text { docentes } \\
\text { con postgrado }\end{array}$ & $\begin{array}{l}\text { Correacion de Pearson } \\
\text { Sig, (biateral) } \\
\mathrm{N}\end{array}$ & $\begin{array}{r}.637^{\prime \prime} \\
.000 \\
52 \\
\end{array}$ & $\begin{array}{r}6,697 " \\
, 000 \\
52 \\
\end{array}$ & $\begin{array}{r}.744^{\prime \prime} \\
.000 \\
51 \\
\end{array}$ & $\begin{array}{r}6,633^{\prime \prime} \\
, 000 \\
51 \\
\end{array}$ & $\begin{array}{r}.874^{\prime \prime} \\
.000 \\
52 \\
\end{array}$ & $\begin{array}{r}.332 \\
, 153 \\
20 \\
\end{array}$ & $\begin{array}{l}.913^{\prime \prime} \\
.000 \\
.0\end{array}$ & $\begin{array}{l}, 497 \\
.042 \\
\end{array}$ & $\begin{array}{l}9766^{\prime \prime} \\
.000 \\
.6\end{array}$ & $\begin{array}{r}9.980^{\circ} \\
.000 \\
52 \\
\end{array}$ & 1 & $\begin{array}{r}8,891^{+} \\
, 000 \\
52 \\
\end{array}$ & $\begin{array}{l}212 \\
.132 \\
\end{array}$ & $\begin{array}{r}, 550^{\prime \prime} \\
, 000 \\
52 \\
\end{array}$ & $\begin{array}{l}.578^{\prime \prime} \\
.000\end{array}$ & $\begin{array}{l}6,645^{\prime \prime} \\
, 000\end{array}$ \\
\hline $\begin{array}{l}\text { Total Proyectos } \\
\text { Fondecy } \\
\text { untimos } 3 \text { años }\end{array}$ & $\begin{array}{l}\text { Correacón de Pearson } \\
\text { Sig, (biateral) } \\
\mathrm{N}\end{array}$ & \begin{tabular}{rl|}
, $509^{\prime \prime}$ \\
, 000 \\
53
\end{tabular} & $\begin{array}{r}.543^{\prime \prime} \\
.000 \\
53 \\
\end{array}$ & $\begin{aligned} 6,690^{\circ} \\
, 000 \\
52\end{aligned}$ & $\begin{array}{r}.6277^{\circ} \\
, 000 \\
52 \\
\end{array}$ & $\begin{array}{l}.966 " \\
.000 \\
53\end{array}$ & $\begin{array}{l}.284 \\
.226 \\
20\end{array}$ & \begin{tabular}{rl|}
, $979 "$ \\
.000 \\
53
\end{tabular} & $\begin{array}{r}.411 \\
.101 \\
17\end{array}$ & $\begin{array}{r}8.873 " \\
.000 \\
52 \\
\end{array}$ & $\begin{array}{r}.926 " \\
, 000 \\
52 \\
52\end{array}$ & \begin{tabular}{|l|}
$.891 "$ \\
.000 \\
52 \\
\end{tabular} & 1 & $\begin{array}{r}.246 \\
.075 \\
53 \\
\end{array}$ & $\begin{array}{r}, 742 " \\
, 000 \\
53 \\
\end{array}$ & \begin{tabular}{rl|}
, $727 "$ \\
.000 \\
53
\end{tabular} & $\begin{aligned}, 784^{4} \\
, 000 \\
52\end{aligned}$ \\
\hline 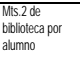 & $\begin{array}{l}\text { Conereación de Pearson } \\
\text { sig. (bilteral) } \\
N\end{array}$ & $\begin{array}{l}, 347^{\circ} \\
.011 \\
53\end{array}$ & $\begin{array}{l}, 294 \\
, 033 \\
53 \\
\end{array}$ & $\begin{array}{l}.064 \\
, 654 \\
52\end{array}$ & $\begin{array}{l}, 157 \\
, 266 \\
52 \\
\end{array}$ & $\begin{array}{r}.212 \\
, 128 \\
53\end{array}$ & $\begin{array}{l}.421 \\
.065 \\
20\end{array}$ & $\begin{array}{r}.226 \\
.104 \\
53\end{array}$ & $\begin{array}{r}-410 \\
, 102 \\
17\end{array}$ & \begin{tabular}{r|}
.233 \\
.096 \\
52
\end{tabular} & , 240 & \begin{tabular}{r|}
.212 \\
.132 \\
52 \\
\end{tabular} & , 246 & 1 & \begin{tabular}{r|r}
, $453^{\prime \prime}$ \\
, 001 \\
53 \\
\end{tabular} & $\begin{array}{r}, 109 \\
, 437 \\
53\end{array}$ & $\begin{array}{l}1,167 \\
, 232\end{array}$ \\
\hline $\begin{array}{l}\mathbb{N}^{N} \text { volumenes } \\
\text { por a lumnos }\end{array}$ & 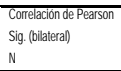 & $\begin{array}{ll}.374^{\prime \prime} \\
.006 \\
53\end{array}$ & $\begin{array}{r}.360 " \\
.009 \\
53 \\
53\end{array}$ & $\begin{array}{r}.304^{\circ} \\
.029 \\
52\end{array}$ & \begin{tabular}{r|}
.317 \\
.022 \\
52 \\
\end{tabular} & $\begin{array}{l}.733^{\prime \prime} \\
.000 \\
53\end{array}$ & $\begin{array}{r}.403 \\
.078 \\
20\end{array}$ & $\begin{aligned} .703^{\prime \prime} \\
.000 \\
53\end{aligned}$ & $\begin{array}{r}-.137 \\
.601 \\
17 \\
17\end{array}$ & $\begin{array}{r}5377^{\circ} \\
.000 \\
52 \\
\end{array}$ & $\begin{array}{r}.610^{6} \\
.000 \\
52 \\
5\end{array}$ & $\begin{array}{r}.550^{\circ \prime} \\
.000 \\
52 \\
\end{array}$ & $\begin{array}{r}7,742^{\circ} \\
, 000 \\
53\end{array}$ & $\begin{array}{r}.453^{\prime \prime} \\
, 001 \\
53 \\
\end{array}$ & $\begin{array}{r}1 \\
53 \\
\end{array}$ & $\begin{aligned}, 536 " \\
.000 \\
53\end{aligned}$ & $\begin{array}{l}.608^{\prime \prime} \\
.000 \\
53\end{array}$ \\
\hline $\begin{array}{l}\text { Mist.2 2de } \\
\text { laboratarios }\end{array}$ & $\begin{array}{l}\text { Corelacón de Pearson } \\
\text { Sig. (bilatera) } \\
\mathrm{N}\end{array}$ & $\begin{array}{r}.306^{\circ} \\
.026 \\
53 \\
\end{array}$ & $\begin{array}{r}.319^{\circ} \\
.020 \\
53 \\
\end{array}$ & $\begin{array}{r}.511^{\prime \prime} \\
.000 \\
52 \\
\end{array}$ & $\begin{array}{r}.497 " \\
.000 \\
52 \\
\end{array}$ & $\begin{array}{l}.716^{\prime \prime} \\
.000 \\
53 \\
5\end{array}$ & $\begin{array}{r}.309 \\
.186 \\
20 \\
\end{array}$ & $\begin{array}{r}.671 " \\
.000 \\
53 \\
\end{array}$ & $\begin{array}{r}.308 \\
, 229 \\
17 \\
\end{array}$ & $\begin{array}{r}.602 " \\
.000 \\
52 \\
\end{array}$ & $\begin{array}{r}, 574 " \\
, 000 \\
52 \\
\end{array}$ & \begin{tabular}{|l|}
, $578^{" \prime}$ \\
.000 \\
52 \\
\end{tabular} & \begin{tabular}{|c|}
, $727^{\prime \prime}$ \\
, 000 \\
53 \\
\end{tabular} & \begin{tabular}{rl|}
, 109 \\
.437 \\
53 \\
\end{tabular} & $\begin{array}{r}.536 " \\
, 000 \\
53 \\
\end{array}$ & 1 & $\begin{array}{r}.879^{-1} \\
.000 \\
53 \\
\end{array}$ \\
\hline $\begin{array}{l}\text { Node } \\
\text { laboratorios }\end{array}$ & $\begin{array}{l}\text { Correlacón de Pearson } \\
\text { Sig. (bilatera) } \\
\mathrm{N}\end{array}$ & $\begin{array}{r}, 371^{\prime \prime} \\
.006 \\
53 \\
\end{array}$ & $\begin{array}{r}.363 " \\
.008 \\
53 \\
\end{array}$ & $\begin{array}{r}., 521^{\prime \prime} \\
.000 \\
52 \\
\end{array}$ & $\begin{array}{r}.485 " \\
.000 \\
52 \\
\end{array}$ & $\begin{array}{l}, 743^{\prime \prime} \\
.000 \\
53 \\
\end{array}$ & $\begin{array}{r}.358 \\
, 121 \\
20 \\
\end{array}$ & $\begin{array}{l}.735^{\prime \prime} \\
.000 \\
53 \\
\end{array}$ & $\begin{array}{r}.342 \\
, 180 \\
17 \\
\end{array}$ & $\begin{array}{r}.653 " \\
.000 \\
52 \\
\end{array}$ & $\begin{array}{r}.657 " \\
.000 \\
52 \\
\end{array}$ & \begin{tabular}{|c|}
, $645^{\prime \prime}$ \\
.000 \\
52 \\
\end{tabular} & $\begin{array}{r}7,784 \\
, 000 \\
53 \\
\end{array}$ & \begin{tabular}{rl|}
, 167 \\
.232 \\
53 \\
\end{tabular} & $\begin{array}{r}, 6088^{\prime \prime} \\
, 000 \\
53 \\
\end{array}$ & $\begin{array}{r}8,879^{\prime \prime} \\
, 000 \\
53 \\
\end{array}$ & 1 \\
\hline 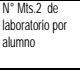 & $\begin{array}{l}\text { Correaciononde } \\
\text { sig. (biatera) } \\
\mathrm{N}\end{array}$ & $\begin{array}{r}, 172 \\
.217 \\
53 \\
\end{array}$ & $\begin{array}{r}, 205 \\
, 141 \\
53\end{array}$ & $\begin{array}{r}, 235 \\
, 094 \\
52 \\
\end{array}$ & $\begin{array}{r}3577^{\prime \prime} \\
.009 \\
52 \\
\end{array}$ & $\begin{array}{r}.266 \\
.054 \\
53\end{array}$ & $\begin{array}{r}., 42 \\
, 051 \\
20\end{array}$ & $\begin{array}{r}, 232 \\
.094 \\
53 \\
\end{array}$ & $\begin{array}{r}.081 \\
, 757 \\
17\end{array}$ & $\begin{array}{l}, 203 \\
, 149\end{array}$ & $\begin{array}{r}.210 \\
, 135 \\
52 \\
\end{array}$ & $\begin{array}{r}198 \\
, 160 \\
52 \\
\end{array}$ & $\begin{array}{r}, 264 \\
, 056 \\
53\end{array}$ & $\begin{array}{r}.097 \\
.490 \\
53\end{array}$ & $\begin{array}{r}, 195 \\
, 161 \\
53 \\
5\end{array}$ & $\begin{array}{r}, 669^{\prime \prime} \\
, 000 \\
53 \\
\end{array}$ & $\begin{array}{l}, 456 " \\
, 001\end{array}$ \\
\hline
\end{tabular}


Anexo 4. Prueba T-Student para diferencia de medias de indicadores de acreditación según tipo de Universidad

Estadísticos de grupo

\begin{tabular}{|c|c|c|c|c|c|}
\hline & $\begin{array}{l}\text { Tipo de Universidades } \\
\text { según Origen }\end{array}$ & $\mathrm{N}$ & Media & Desviación típ. & $\begin{array}{l}\text { Error típ. de la } \\
\text { media }\end{array}$ \\
\hline \multirow{2}{*}{$\begin{array}{l}\mathrm{N}^{\circ} \text { de años de acreditación } \\
\text { institucional }\end{array}$} & Consejo de Rectores & 25 & 4,40 & 1,756 & ,351 \\
\hline & Privada & 28 & 2,68 & 2,001 & ,378 \\
\hline \multirow[t]{2}{*}{ Número de áreas acreditadas } & Consejo de Rectores & 25 & 3,40 & 1,323 &, 265 \\
\hline & Privada & 28 & 1,68 & 1,219 & ,230 \\
\hline \multirow[t]{2}{*}{ Número de carreras acreditadas } & Consejo de Rectores & 24 & 16,2917 & 10,61778 & 2,16735 \\
\hline & Privada & 28 & 7,2500 & 4,06088 & ,76743 \\
\hline \multirow{2}{*}{$\begin{array}{l}\mathrm{N}^{\circ} \text { de programas de magíster } \\
\text { acreditados }\end{array}$} & Consejo de Rectores & 25 & 6,64 & 12,315 & 2,463 \\
\hline & Privada & 28 &, 18 &, 772 & , 146 \\
\hline \multirow{2}{*}{$\begin{array}{l}N^{\circ} \text { de programas de doctorado } \\
\text { acreditados }\end{array}$} & Consejo de Rectores & 25 & 4,88 & 9,171 & 1,834 \\
\hline & Privada & 28 & 21 &, 630 &, 119 \\
\hline \multirow{2}{*}{$\begin{array}{l}\text { Promedio años de acreditación de } \\
\text { carreras }\end{array}$} & Consejo de Rectores & 24 & 3,87389 & 1,043685 & ,213041 \\
\hline & Privada & 28 & 3,23088 & 894736 & 169089 \\
\hline
\end{tabular}

\begin{tabular}{|c|c|c|c|c|c|c|c|c|c|c|}
\hline \multicolumn{11}{|c|}{ Prueba de muestras independientes } \\
\hline & & \multicolumn{2}{|c|}{$\begin{array}{l}\text { Prueba de Levene para la } \\
\text { igualdad de varianzas }\end{array}$} & \multicolumn{7}{|c|}{ Prueba T para la igualdad de medias } \\
\hline & & \multirow[b]{2}{*}{$\mathrm{F}$} & \multirow[b]{2}{*}{ Sig. } & \multirow[b]{2}{*}{$\mathrm{t}$} & \multirow[b]{2}{*}{ gl } & \multirow[b]{2}{*}{ Sig. (bilateral) } & \multirow{2}{*}{$\begin{array}{l}\text { Diferencia de } \\
\text { medias }\end{array}$} & \multirow{2}{*}{$\begin{array}{l}\text { Error típ. de la } \\
\text { diferencia }\end{array}$} & \multicolumn{2}{|c|}{$\begin{array}{c}95 \% \text { Intervalo de confianza para la } \\
\text { diferencia }\end{array}$} \\
\hline & & & & & & & & & Inferior & Superior \\
\hline $\begin{array}{l}N^{\circ} \text { de años de } \\
\text { acreditación }\end{array}$ & $\begin{array}{l}\text { Se han asumido } \\
\text { varianzas iguales }\end{array}$ & ,988 &, 325 & 3,311 & 51 & ,002 & 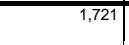 &, 520 & ,678 & 2,765 \\
\hline institucional & $\begin{array}{l}\text { No se han asumido } \\
\text { varianzas iguales }\end{array}$ & & & 3,336 & 50,988 &, 002 & 1,721 &, 516 & 685 & 2,757 \\
\hline $\begin{array}{l}\text { Número de áreas } \\
\text { acreditadas }\end{array}$ & $\begin{array}{l}\text { Se han asumido } \\
\text { varianzas iguales } \\
\text { No se han asumido } \\
\text { varianzas iguales }\end{array}$ & .418 &, 521 & $\begin{array}{l}4,931 \\
4,907\end{array}$ & $\begin{array}{r}51 \\
49,097\end{array}$ & $\begin{array}{l}, 000 \\
.000\end{array}$ & $\begin{array}{l}1,721 \\
1,721\end{array}$ & $\begin{array}{l}.349 \\
, 351\end{array}$ & \begin{tabular}{l|}
1,021 \\
1,017
\end{tabular} & $\begin{array}{l}2,422 \\
2,426\end{array}$ \\
\hline $\begin{array}{l}\text { Número de } \\
\text { carreras } \\
\text { acreditadas }\end{array}$ & $\begin{array}{l}\text { Se han asumido } \\
\text { varianzas iguales } \\
\text { No se han asumido } \\
\text { varianzas iguales }\end{array}$ & 22,762 & ,000 & $\begin{array}{l}4,170 \\
3,933\end{array}$ & $\begin{array}{r}50 \\
28,744\end{array}$ & $\begin{array}{l}, 000 \\
, 000\end{array}$ & $\begin{array}{l}9,04167 \\
9,04167\end{array}$ & $\begin{array}{l}2,16841 \\
2,29920\end{array}$ & $\begin{array}{l}4,68629 \\
4,33745\end{array}$ & $\begin{array}{l}13,39704 \\
13,74589\end{array}$ \\
\hline $\begin{array}{l}N^{\circ} \text { de programas } \\
\text { de magíster } \\
\text { acreditados }\end{array}$ & $\begin{array}{l}\text { Se han asumido } \\
\text { varianzas iguales } \\
\text { No se han asumido } \\
\text { varianzas iguales }\end{array}$ & $\begin{array}{ll}15,086 \\
\end{array}$ &, 000 & $\begin{array}{l}2,774 \\
2,619\end{array}$ & \begin{tabular}{r|}
51 \\
24,169
\end{tabular} & \begin{tabular}{l|}
, 008 \\
015 \\
, 015
\end{tabular} & \begin{tabular}{l|}
6,461 \\
6,461
\end{tabular} & $\begin{array}{l}2,330 \\
2,467\end{array}$ & \begin{tabular}{l|}
1,784 \\
1,371
\end{tabular} & $\begin{array}{l}11,138 \\
11,552\end{array}$ \\
\hline $\begin{array}{l}N^{\circ} \text { de programas } \\
\text { de doctorado } \\
\text { acreditados }\end{array}$ & $\begin{array}{l}\text { Se han asumido } \\
\text { varianzas iguales } \\
\text { No se han asumido } \\
\text { varianzas iguales }\end{array}$ & $\begin{array}{ll}19,680 \\
\end{array}$ & ,000 & $\begin{array}{l}2,688 \\
2,538\end{array}$ & $\begin{array}{r}51 \\
24,202 \\
\end{array}$ & \begin{tabular}{l|}
, 010 \\
, 018
\end{tabular} & $\begin{array}{l}, 666 \\
4,666\end{array}$ & $\begin{array}{l}1,736 \\
1,838\end{array}$ & \begin{tabular}{r|}
1,181 \\
, 874
\end{tabular} & $\begin{array}{l}8,150 \\
8,458\end{array}$ \\
\hline $\begin{array}{l}\text { Promedio años de } \\
\text { acreditación de } \\
\text { carreras }\end{array}$ & $\begin{array}{l}\text { Se han asumido } \\
\text { varianzas iguales } \\
\text { No se han asumido } \\
\text { varianzas iguales }\end{array}$ & .505 & ,481 & $\begin{array}{l}2,393 \\
2,364\end{array}$ & $\begin{array}{r}50 \\
45,667\end{array}$ & \begin{tabular}{l|}
.021 \\
.022
\end{tabular} & $\begin{array}{l}, 643012 \\
, 643012\end{array}$ & $\begin{array}{l}, 268747 \\
271989\end{array}$ & \begin{tabular}{l|}
.103219 \\
.095420
\end{tabular} & $\begin{array}{l}1,182806 \\
1,190605\end{array}$ \\
\hline
\end{tabular}

Anexo 5. Test Chi-cuadrado: Tipo de universidad y acreditación del área de investigación

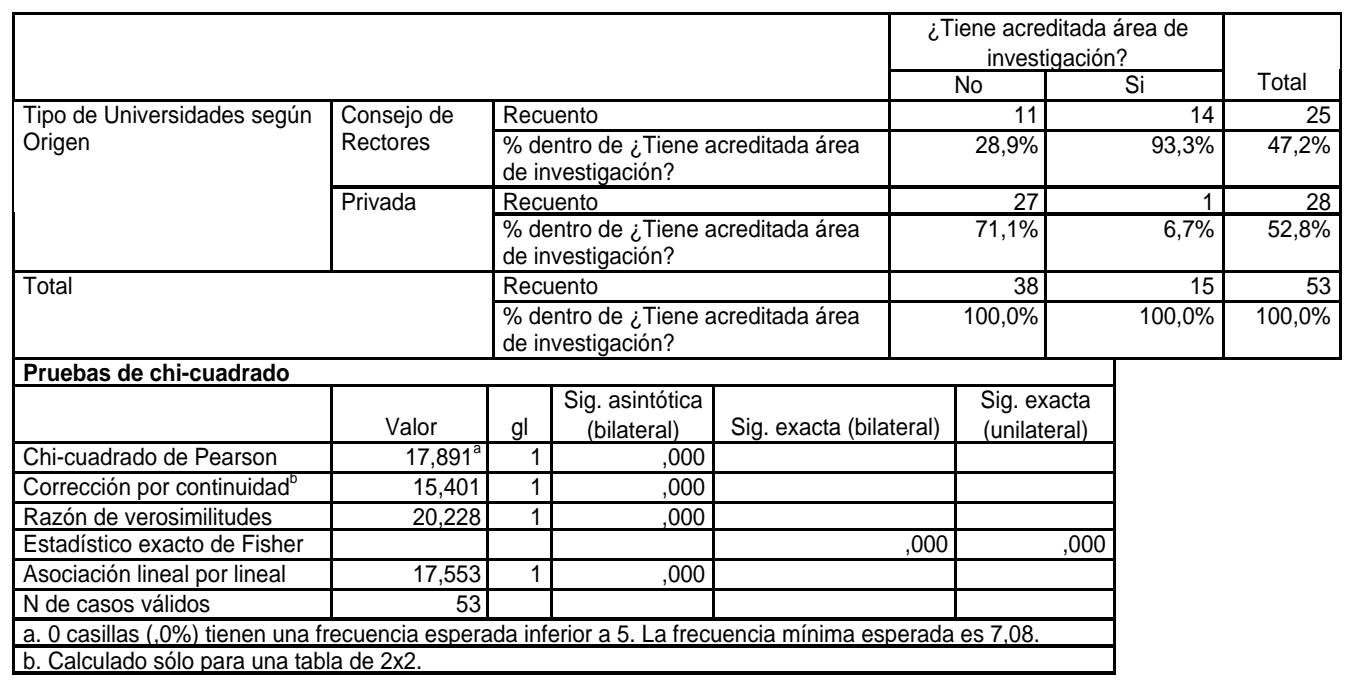


Anexo 6. Test Chi-cuadrado: Tipo de universidad y acreditación del área de vinculación con el medio

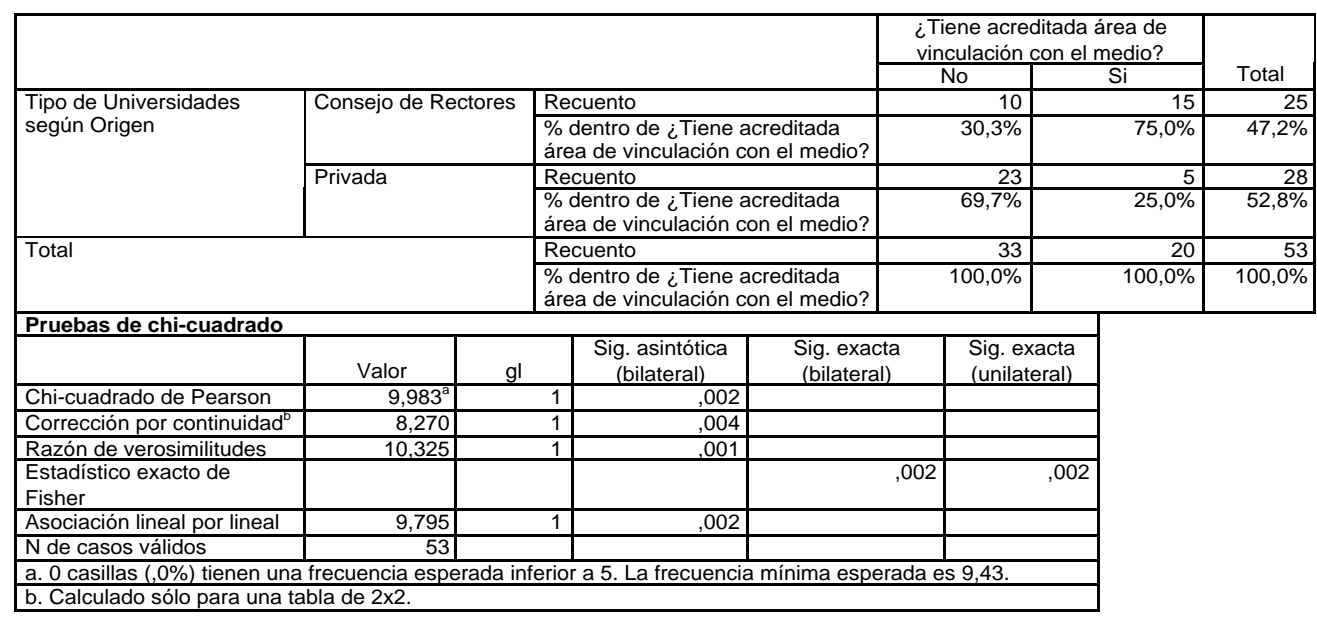

Anexo 7. Test Chi-cuadrado: Ubicación geográfica universidad y acreditación del área de vinculación con el medio

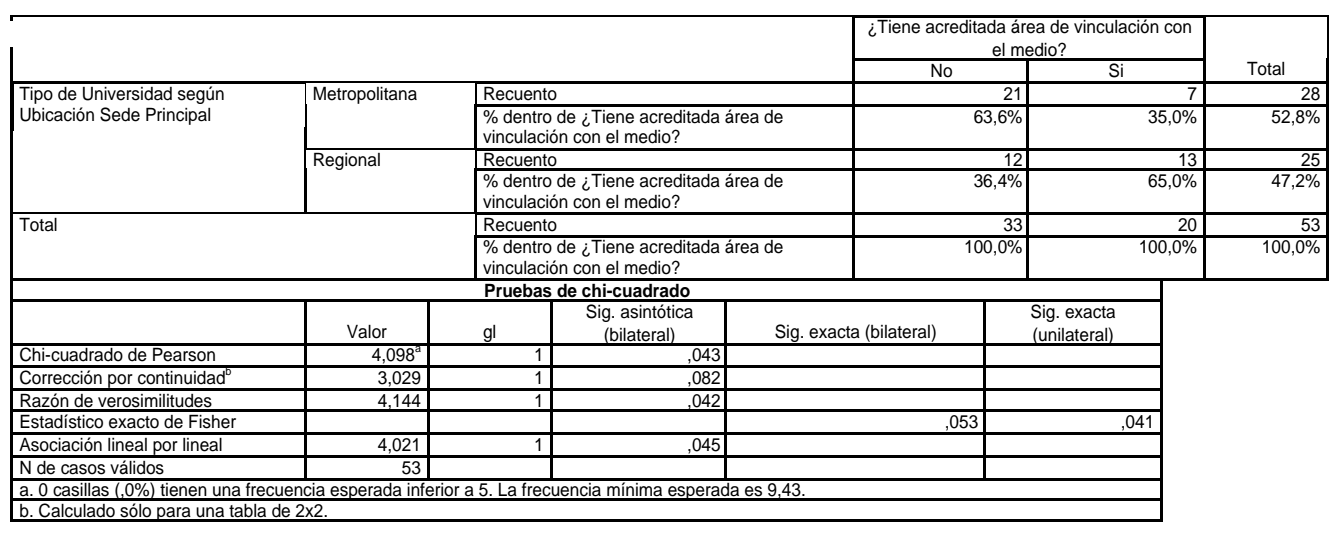




\section{REFERENCIAS BIBLIOGRÁFICAS}

Bernasconi, A. y Rojas, F. (2003). Informe sobre la educación superior en Chile: 1980-2002. IESALC-UNESCO.

Brunner, J.J. (2007). Mercados universitarios: los nuevos escenarios de la educación superior. Informe Final de Proyecto FONDECYT N 1050138.

CNA (2010). Información de acreditación institucional, programas de pregrado y postgrado. Comisión Nacional de Acreditación. Disponible en www.cnachile.cl, consultado el 15/11/2010.

CNED (2010a). Sistema de Información: INDICES 2011, Consejo Nacional de Educación.

CNED (2010b). Acreditación: INDICES 2011, Consejo Nacional de Educación.

Cruz-Coke, R. (2004). Evolución de las universidades chilenas 1981-2004. Revista Médica de Chile, vol 132, n. 12, pp 1543-49, diciembre 2004, Santiago-Chile,

Espinoza, Ó. et al. (2006). Informe: educación superior en Iberoamérica el caso de Chile. Centro Interuniversitario de Desarrollo CINDA.

LGE (2010). Ley General de Educación. Disponible en Biblioteca del Congreso Nacional de Chile, en www.bcn.cl, consultado el 30-11-2010.

MINEDUC (2010). Evolución de la Matrícula de Educación Superior en Chile. Periodo 1990-2009. Santiago de Chile: División de Educación Superior, Ministerio de Educación.

MINEDUC (s/f). Resultado del Primer Ciclo de Acreditación Institucional. Santiago: Ministerio de Educación de Chile. Disponible en: http://www.mineduc.cl/biblio/, consultado el 20/11/2010.

OCDE (2009). La Educación Superior en Chile. Santiago de Chile: OCDE-Banco Mundial.

Ugarte, J. L. (2010). Lineamientos de la Política de Educación Superior: Desafíos y Oportunidades. En Jornada de Reflexión Académica Plan Estratégico 2015. Curicó: Universidad de Talca.

Zapata, G. (2004). Acreditación institucional en Chile: una opción emergente. Revista Calidad en la Educación. El aseguramiento de la calidad de la educación superior, vol. 21, n. 2. 